\title{
Reweighted nuclear PDFs using heavy-flavor production data at the LHC
}

\author{
Aleksander Kusina $\odot,{ }^{1}$ Jean-Philippe Lansberg $\odot,{ }^{2}$ Ingo Schienbein, ${ }^{3}$ and Hua-Sheng Shao $\odot^{4}$ \\ ${ }^{1}$ Institute of Nuclear Physics, Polish Academy of Sciences, \\ ul. Radzikowskiego 152, 31-342 Cracow, Poland \\ ${ }^{2}$ Université Paris-Saclay, CNRS, IJCLab, 91405 Orsay, France \\ ${ }^{3}$ Laboratoire de Physique Subatomique et de Cosmologie, Université Grenoble-Alpes, \\ CNRS/IN2P3, 53 avenue des Martyrs, 38026 Grenoble, France \\ ${ }^{4}$ Laboratoire de Physique Théorique et Hautes Energies (LPTHE), UMR 7589, \\ Sorbonne Université et CNRS, 4 place Jussieu, 75252 Paris, France
}

(Received 8 January 2021; revised 20 April 2021; accepted 19 May 2021; published 13 July 2021)

\begin{abstract}
We present the reweighting of two sets of nuclear parton distribution functions (PDFs), nCTEQ15 and EPPS16, using a selection of experimental data on heavy-flavor meson $\left[D^{0}, J / \psi, B \rightarrow J / \psi\right.$ and $\left.\Upsilon(1 S)\right]$ production in proton-lead collisions at the LHC which were not used in the original determination of these nuclear PDFs. The reweighted PDFs exhibit significantly smaller uncertainties thanks to these new heavyflavor constraints. We present a comparison with another selection of data from the LHC and relativistic heavy ion collider (RHIC) which were not included in our reweighting procedure. The comparison is overall very good and serves as a validation of these reweighted nuclear PDF sets, which we dub nCTEQ15 ${ }_{\text {rwHF }}$ and

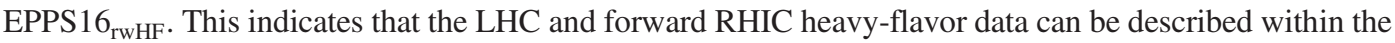
standard collinear factorization framework with the same (universal) small- $x$ gluon distribution. We discuss how we believe such reweighted PDFs should be used as well as the limitations of our procedure.
\end{abstract}

DOI: 10.1103/PhysRevD.104.014010

\section{INTRODUCTION}

Nuclear parton distributions (nPDFs) are important tools for studying heavy-ion high-energy collisions and learn about the partonic structure of nuclei. In particular, our knowledge of the nuclear gluon distribution is crucial to disentangle initial- and final-state effects in nucleusnucleus collisions producing a quark-gluon plasma at the LHC or RHIC.

Regarding the constraints on the nuclear gluon distribution, specific data sets are added to the fits for this purpose. Yet, all of them show limitations, e.g., a limited kinematic coverage in the longitudinal momentum fraction, $x$. First, let us mention the digital integration system (DIS) data from the NMC experiment [1] which allow one to probe nPDFs in the range $10^{-2} \lesssim x \lesssim 0.7$. However, their constraints on the gluon distribution are not direct as they come through evolution effects and thus depend much on the lever arm in the scale. Second, the $W$ and $Z$ boson data from $p \mathrm{~Pb}$ collisions at the LHC [2-7] provide constraints on gluon nPDFs, but only for $x \gtrsim 10^{-3}$. These are also

Published by the American Physical Society under the terms of the Creative Commons Attribution 4.0 International license. Further distribution of this work must maintain attribution to the author(s) and the published article's title, journal citation, and DOI. Funded by SCOAP. indirect, as the quark-gluon channels enter only at next-toleading order (NLO). Nevertheless, they probably provide the cleanest available probe of the nuclear gluon in this $x$ domain. Third, the $p \mathrm{~Pb}$ di-jet data from the CMS experiment [8], used by the EPPS16 analysis [9], are promising as they are directly sensitive to the gluon nPDF. However, they give constraints only for $x \gtrsim 10^{-2}$, and our current understanding of these data is questionable (see Sec. IV F).

In view of the above, it is clear that we still lack solid constraints on the small- $x$ gluon nuclear distribution which are, among other things, very important to understand the phenomenon of gluon saturation. Among the currently available experimental data which have not yet been included in nPDF fits, we can cite: isolated photon production, semi-inclusive hadron $(\mathrm{SIH})$ production from LHC, top-quark production and obviously heavy-flavor (HF) production all from $p \mathrm{~Pb}$ collisions at the LHC, which is at the center of our analysis.

Yet, all these data show limitations. The currently available LHC isolated-photon data [10] taken in $p \mathrm{~Pb}$ collisions in Run II at $\sqrt{s_{\mathrm{NN}}}=8.16 \mathrm{TeV}$ are given as a function of the transverse energy $\left(E_{T}^{\gamma}\right)$ of the photon in the range $E_{T}^{\gamma} \in[20,550] \mathrm{GeV}$ for three bins at central rapidity. Assuming leading order kinematics this roughly corresponds to $x \gtrsim 5 \times 10^{-4}$. Recent attempts to include this data set in a global fit [11] gave a poor $\chi^{2} /$ point $\sim 6$. In particular, the region with the smallest $E_{T}^{\gamma}$ (and hence 
smaller $x$ ) is where the nondirect contribution, depending on the photon fragmentation function, is largest. In the case of the SIH data, e.g., [12,13], the most serious issue arises from the strong dependence on the fragmentation functions which are not well known. Additionally the final-state effects, like energy loss, might be of importance. Furthermore, if one focuses on the smallest $x$, the SIH data set would be restricted to small $P_{T}$ where perturbative QCD (pQCD) computations may not be completely under control. LHC top-quark data would constrain well the large- $x$ gluon distribution but the current data from $p \mathrm{~Pb}$ collisions remain of limited precision and are not differential, which limits the possible constraints on the nPDFs. Finally, the HF data, such as those we used in [14], are unique as they give access to very small- $x$ values, down to $10^{-5}$. They are also not free from theoretical limitations. Quarkonium production is admittedly not well understood with different mechanisms proposed to explain it (see e.g., [15-17]) and it is thus not clear whether the produced heavy-quark pair is colorless or not when it crosses the remnants of the colliding nucleus [16]. On the other hand, one-particle inclusive open heavy-flavor production is theoretically well understood and measurements of heavy-flavored meson and baryon production by ALICE, CMS, ATLAS, and LHCb are generally in reasonable agreement with the theoretical predictions by FONLL and GM-VFNS within the large scale uncertainties [16]. Nevertheless, heavy-quark data from $p \mathrm{~Pb}$ collisions at the LHC have not (yet) been included in a global fit of nPDFs. Even though all the data sets mentioned in this introduction can provide complementary constraints on gluon nPDFs, HF production appear to us as the most promising one to provide, right now, useful constraints for studies where gluon nPDFs are used at small $x$, before new fits using new gluon constraints are available.

The very low- $x$ nuclear gluon distribution will remain very hard to constrain even with the possible future experiments. The most promising experiment in this regard - the electron-ion collider (EIC)—will allow one to probe nPDFs only down to $x \gtrsim 5 \times 10^{-4}[18,19]$. This makes the HF $p \mathrm{~Pb}$ LHC data even more unique and worth the additional effort for their understanding and inclusion in nPDF fits.

In [14], we have thus investigated how experimental data on the inclusive production of HF mesons $\left[D^{0}, J / \psi, B \rightarrow\right.$ $J / \psi$ and $\Upsilon(1 S)]$ in proton-lead collisions at the LHC could advance our knowledge of the gluon nPDF of heavy nuclei. Indeed, at the LHC and RHIC energies, such HF reactions are usually initiated by gluons and their production in proton-nucleus collisions should shed some light on the nuclear gluon content. ${ }^{1}$

\footnotetext{
${ }^{1}$ Recently, associated HF reactions were also shown [20,21] to be good probes of the impact-parameter-dependent partonic nuclear content.
}

We observed that the nuclear effects encoded in two recent global fits of nuclear parton densities at NLO, nCTEQ15 [22] and EPPS16 [9], were yielding a good description of the existing LHC HF production data supporting the hypothesis that the modification of the partonic densities in heavy nuclei could be the dominant effect at play in such processes.

We then went further in our investigations and performed a Bayesian-reweighting analysis of the data sample of each of the mesons and showed that the existing HF data were clearly pointing at a depleted gluon distribution at small momentum fractions, $x$, in the lead nucleus, also known as shadowing. According to our reweighting analysis, the significance of this depletion was larger than seven $\sigma$ at $x$ smaller than 0.01. In addition, our analysis also supported the existence of gluon antishadowing, whereby the gluon content of nuclei is augmented when $x \simeq 0.1$.

We concluded that the inclusion of such HF data in a nPDF fit, such as those used for our analysis, would reduce the uncertainty on the gluon density in heavy nuclei down to $x \simeq 7 \times 10^{-6}$. At such low $x$, there is currently simply no other data as we have just discussed. We stressed that the reweighted nPDFs would still be compatible with the other data of the global fits, in particular in the quark sector.

Yet, we noted that the large factorization scale $\left(\mu_{F}\right)$ uncertainty in the computation of HF-production cross sections and nuclear modification factors (NMFs) should be fully taken into consideration, in particular in the charm sector. We indeed found it to generate an uncertainty larger than that encapsulated in nPDF sets reweighted using these charm or charmonium data. Indeed, the magnitude of the nuclear effects encoded in the nPDFs depends on the scale via the Dokshitzer-Gribov-Lipatov-Altarelli-Parisi (DGLAP) evolution equation [23-25]. Such an evolution is admittedly fast in the $\mathrm{GeV}$ range and the reweighted nPDFs depend much on the value of the scale where the data contraints are set if it happens to be near twice the charm mass.

Since our study [14], only a couple of new data sets applicable to global nPDF analyses have appeared, e.g., the Run II CMS $W^{ \pm}$data [2] or DIS data from JLAB [26]. On the theory side, a number of new determinations of nPDFs from various groups $[11,27-30]$ became available. These new determinations brought in some new developments. In particular, they advanced the nPDF analyses to the next-tonext-to-leading (NNLO) order of QCD. ${ }^{2}$ Another notable fact was the first use of Monte-Carlo based determination of uncertainties in the context of nPDFs [11]. However, none of them superseded our HF reweighting study.

In this context, we follow up on our previous study [14] published as a Letter. First, we provide more details about the reweighting analysis and new comparisons with data

\footnotetext{
${ }^{2}$ One should note that the current NNLO analyses are using only certain types of data, in most cases these are only DIS data.
} 
sets which became available after our study was performed. They serve as post validation of our reweighting analysis. Second we have converted our reweighted nPDFs, which were initially in the form of replicas along with weights, into nPDFs with Hessian uncertainties. As such, they can be presented in files to be included and easily used in the LHAPDF library. A new feature of our analysis is that it includes the effect of the factorization-scale choice. Third, we provide specific guidances on how to use them for future studies, in particular to predict the initialstate nuclear effect on hard probes of the quark-gluon plasma (QGP).

\section{CONTEXT AND METHODOLOGY}

\section{A. Framework and its justification}

In the collinear factorization, following Feynman's model of partons, the longitudinal-momentum distributions of quarks and gluons inside hadrons are given by the PDFs. These then connect the measurable hadronic cross sections and the partonic cross sections (induced by quarks and gluons) which can be calculated using perturbative methods.

The determination of PDFs of free protons, denoted $f_{i}^{p}$, is actively pursued by many colleagues via global analyses of as much as possible experimental data of hard processes which are believed to be perturbative enough. Such state of the art global analyses [31-36] involve complex perturbative calculations and cutting-edge statistical methods to extract PDFs and their uncertainties altogether.

Assuming the same collinear factorization to apply in proton-nucleus $(p A)$ and nucleus-nucleus $(A A)$ collisions, one needs to introduce nPDFs, denoted $f_{i}^{A}$. In such a case, additional challenges come out along with additional motivations to study them. Indeed, nuclear data are significantly more complex to collect and one needs to cope with two additional degrees of freedom to describe the nuclei, the number of protons $(Z)$ and neutrons $(N=A-Z)$ which they comprise. These are also necessary inputs to employ hard probes of the QGP produced in ultra-relativistic heavy-ion collisions at RHIC and the LHC [16]. Constraining nPDFs is then not only about the ambitious endeavor to understand the quark and gluon contents of the nuclei but also to understand the initial stages of the production of some QGP probes.

Since the early 1980's, we know that the partonic description of nuclei cannot be reduced to a simple collection of partons in free nucleons. In other words, nPDFs deviate from a simple sum of nucleon PDFs. To study such deviations, it is customary to rely on NMFs, like

$$
R\left[F_{2}^{\ell A}\right]=\frac{F_{2}^{\ell A}}{Z F_{2}^{\ell p}+N F_{2}^{\ell n}}
$$

for the DIS structure function $F_{2}$ and parton-level NMFs

$$
R_{i}^{A}\left(x, \mu_{F}\right)=\frac{f_{i}^{A}}{Z f_{i}^{p}+N f_{i}^{n}}
$$

with $f_{i}^{A} \equiv Z f_{i}^{p / A}+N f_{i}^{n / A}$, instead of the absolute nPDFs. Past studies of $F_{2}$ [37-43] told us that, for the quarks,

(i) $R_{q}^{A}>1$ for $x \gtrsim 0.8$ (Fermi-motion region),

(ii) $R_{q}^{A}<1$ for $0.25 \lesssim x \lesssim 0.8$ (EMC region),

(iii) $R_{q}^{A}>1$ for $0.1 \lesssim x \lesssim 0.25$ (antishadowing region), and

(iv) $R_{q}^{A}<1$ for $x \lesssim 0.1$ (shadowing region).

These four different $x$ regions are usually denoted by the names in parenthesis. The EMC region still lacks a fully conclusive picture [44] although, in the region of medium and large $x, R_{q}^{A}$ is usually explained by nuclear-binding and medium effects and the Fermi motion of the nucleons [45]. At small $x$, coherent scatterings inside the nucleus explain the observed suppression of $F_{2}$. This is why it is referred to as shadowing. The physics underlying the antishadowing is however less firmly established.

Although the generic trend of the nPDFs could in principle be related to specific physics phenomena, they remain essentially determined by global fits of experimental data $[9,11,22,27,28,46-48]$ based on initial parametrizations only slightly driven by the above physics considerations.

The above discussion specifically relates to the nuclear quark content which can directly be probed by leptonnucleus $(\ell A)$ DIS and $p A$ Drell-Yan processes. Lacking corresponding direct probes, the nuclear gluon content is less known, despite indirect constraints from the scaling violation [22].

As such, the NLO nPDF fits, nCTEQ15 [22] and EPPS16 [9], were performed using data from RHIC on single inclusive pion production. In the case of EPPS16, jet data from the LHC were also used. The objective was precisely to constrain the gluon densities down to $x \sim 10^{-3}$. However, owing to the absence of data at $x \lesssim 10^{-3}$, the nuclear gluon content remained completely undetermined at small $x$. As such, the resulting gluon nPDFs in this region are mere extrapolations from the region of larger $x$ and essentially follow from their $x$-dependent parametrizations at the scale $\mu_{F, 0} \sim 1 \mathrm{GeV}$ where the perturbative DGLAP evolution is initiated.

As pointed out several times $[49,50]$, the uncertainties derived from these gluon nPDFs is not representative of the true uncertainty on this quantity. More flexible initial nPDF parametrizations naturally yield much larger uncertainties in this region. This explains why the EPPS16 set, despite accounting for more data constraints, show much larger uncertainties with respect to its predecessor EPS09 [51] or nCTEQ15.

In this context and motivated by the results of proton studies using HF production to improve the determination of small- $x$ gluon PDF [52-56], we thus studied [14] the 
impact of HF production to constrain the small- $x$ gluon density in lead down to $x \simeq 7 \times 10^{-6}$. In particular, we used heavy-quark and heavy-quarkonium data in LHC protonlead $(p \mathrm{~Pb})$ collisions.

Like the follow-up work presented here, our former study relied on the assumption that collinear factorization in terms of nPDFs holds in the nuclear environment. To date, the global usage of nPDFs is still a subject of debates. Such an assumption should thus be seen as a working hypothesis which has to be systematically questioned. This is the object of this extension with new data-theory comparisons and the release of reweighted nPDFs which can serve for future studies which will confirm or falsify this framework.

Indeed, we recall that other cold-nuclear matter (CNM) effects [57-73] could be at play in specific conditions, in particular for the quarkonium case. In the adopted framework, such additional effects are however considered as higher-twist (HT) contributions and our working assumption can be seen as the consideration of the sole of the leading-twist (LT) factorizable contributions.

\section{B. Connecting NMFs and nPDFs}

Since the advent of RHIC and the LHC, thus for two decades now, the cross-section measurements performed at $p A$ colliders have nearly systematically been normalized to the $p p$ ones $[16,74,75]$. Indeed, the prime interest of such studies is to look for deviations from the free nucleon case, up to isospin effects.

Hard perturbative reactions are rare and each of the $\left\langle N_{\text {coll. }}\right\rangle$ binary nucleon-nucleon $(\mathrm{NN})$ collisions triggered by a $p A$ or $A A$ collision is meant to independently and equally contribute to the observed yields $Y$ : $Y_{p A / A A} \simeq\left\langle N_{\text {coll. }}\right\rangle Y_{\mathrm{NN}}$. If one considers all the possible geometrical configurations for these collisions, this expected equality can be translated into a relation between the cross sections, namely $d \sigma_{p A} \simeq A \times d \sigma_{p p}$. As such, it is natural to define the NMF

$$
R_{p A} \equiv \frac{d \sigma_{p A}}{A \times d \sigma_{p p}}
$$

such that (up to isospin effects) it would equate unity in the absence of nuclear effects. Just as $R_{q}^{A}$ was defined above, one can define $R_{g}^{A}$ with the difference that gluon densities are a priori identical in protons and neutrons. If this was the only nuclear effect at play, $R_{p A}$ would then directly be connected to $R_{g}^{A}$ via the integration of kinematic variables. Only for specific reactions at leading order can one write an equality. In general, it is necessarily more complex.

In the case of DIS off a nucleus $A$, one historically used $F_{2}^{\ell A}$ instead of $\sigma$ and the corresponding NMF $R\left[F_{2}\right]$ is then naturally found to probe the modification of the (anti)quark nPDF compared to its PDF, i.e., $R_{q}^{A}$ as we discussed above.
The NMFs at the LHC and RHIC are so far differential in the transverse momentum $\left(P_{T, \mathcal{H}}\right)$ or the center-ofmomentum (cms) rapidity $y_{\mathrm{cms}, \mathcal{H}}$ of the observed hadron $\mathcal{H}$ as well as a function of the collision centrality. The latter remains theoretically poorly understood and introduces many complications. As such, it was not considered in [14]. Since the current study relies on this first study, we leave the centrality dependence for future investigations and focus on centrality-integrated results where the geometry of the collisions should not matter.

Focusing on $R_{p A}$ is justified by the following. First, it essentially removes the sensitivity from the theoretical side on the proton PDF. Indeed, at very small $x$, the PDF uncertainties are not necessarily negligible. Second, $R_{p A}$ is a priori less sensitive to the modification of the normalization of the cross-section by QCD corrections to the hard parton scattering. Third, on the experimental side, $R_{p A}$ is usually better determined than the $p A$ yields because some systematic experimental uncertainties can be assumed to cancel.

As we have already alluded to, the connection between $R_{p A}$ and $R_{q, g}^{A}$ is not necessarily trivial even assuming that the reaction is only initiated by gluon fusion as expected at high energy for HF production. Since we follow the procedure of [14], we will employ the data-driven approach of [76-78] where the matrix elements squared $|A|^{2}$ for the gluon-fusion processes are determined from $p p$ data restricting to a $2 \rightarrow 2$ kinematics. ${ }^{3}$ First and foremost, it is justified by our limited understanding of the quarkoniumproduction mechanisms (see e.g., [15-17]) while being sufficient to perform a sound evaluation of the nPDF effects via $R_{p A}$. Second, the same approach also applies to open HF hadrons [76] for which full-fledged pQCD computations also exist ${ }^{4}$ (see below). In practice, it boils down to employ a specific empirical functional form for $|A|^{2}$, initially used in [88] to model single-quarkonium hadroproduction for double parton scattering studies [21,88-93]. The latter is flexible enough to provide a fair account of HF data on single-inclusive-particle production. In fact, for quarkonium production, it happens that complete $\mathrm{pQCD}$ computations, which have however the virtue of being derived from first principles of QCD, are far from being able to describe all the features of the data [15]. Our main motivation in considering quarkonium data is to verify that the constraints set by these data on the gluon nPDF are similar to those set by open-charm and beauty production. It should indeed be the case if our treatment of the kinematics is accurate and the relevant nuclear effects correctly encoded in the nPDFs.

The case of $D$-meson production in $p \mathrm{~Pb}$ collisions has in fact recently been studied in detail [94] using the SACOT- $m_{T}$ variant [95] of the GM-VFNS formalism.

\footnotetext{
${ }^{3}$ See [70] for a discussion of the relevance of a $2 \rightarrow 2$ kinematics to predict $J / \psi R_{p A}$ as a function of $y$.

${ }^{4}$ GM-VFNS [79-83], MG5aMC [84] and FONLL [85-87].
} 


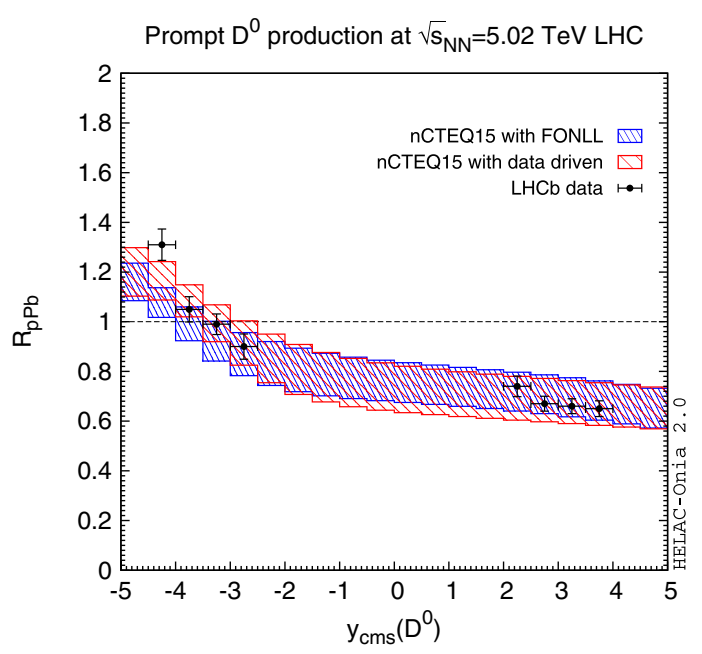

FIG. 1. Comparison between the $D^{0} R_{p \mathrm{~Pb}}$ as a function of $y_{\text {c.m.s. }}$ at $\sqrt{s_{\mathrm{NN}}}=5.02 \mathrm{TeV}$ obtained with nCTEQ15 using FONLL (blue), our data-driven method (red) and LHCb data [96].

It has been found to yield the same qualitative features as what we obtained in [14] regarding the constraints on the nCTEQ15 and EPPS16 nPDFs and the rather large associated factorization-scale uncertainty, despite an apparent difference in the $x$-region involved. In fact, we checked during our initial study that, for $D^{0}$ and $B \rightarrow J / \psi$ production, the scale uncertainty on $R_{p \mathrm{~Pb}}$ obtained with the data-driven approach nearly matches that of FONLL. ${ }^{5}$ This is illustrated by Fig. 1 that shows a comparison of $D^{0} R_{p \mathrm{~Pb}}$ obtained with nCTEQ15 using FONLL and our data-driven method at $\sqrt{s_{\mathrm{NN}}}=5.02 \mathrm{TeV}$. Both approaches indeed show very similar qualitative features except in the backward region where $R_{g}$ quickly varies and where the kinematics probably matters most. Accordingly, we had found that they yield similar reweighting results as we have discussed in a supplemental material to [14]. ${ }^{6}$ The similarity between the reweighting results with FONLL and our data-driven approach is maybe due to the near constant behavior of $R_{g}$ as encoded in nCTEQ15 (before reweighting) at low $x$. Clearly, for a NLO nPDF fit using HF data, it would be more suitable to use a fully fledged pQCD approach. However, for a reweighting study like the one done here where, for charm production, the scale uncertainty would remain the greatest source of uncertainty, this remains surely sufficient.

We would also like to stress that our data-driven approach is effectively a fixed-flavor-number-scheme (FFNS), a threeflavor scheme for charm(onium) to be precise. We find this suitable as the bulk of the proton-nucleus data lies at low $P_{T}$. If we were to treat charm production at $P_{T}$ larger than, say,

\footnotetext{
${ }^{5}$ whereas FONLL shows significantly larger scale uncertainties on the cross section.

${ }^{6}$ See section B and Fig. 1 of https://journals.aps.org/prl/ supplemental/10.1103/PhysRevLett.121.052004/appendix.pdf.
}

$20 \mathrm{GeV}$, it would be justified to take into account the presence of charm quarks in the proton and the associated resummation effects in the charm PDF. At this stage, we cannot exclude that this would lead to a different sensitivity on the probe nPDFs. Yet, we should keep in mind that the charm PDF is strongly correlated to the gluon PDF (see e.g., Fig. 4 in [49]).

We also note that the employed data-driven approach has some additional advantages:

(1) The event generation is much faster than using NLO QCD-based codes. This allows us to perform computations for several nPDFs (two in our case) with three scale choices for four particles in an acceptable amount of computing time.

(2) One can employ it directly for any single-inclusiveparticle spectrum once we know the relative contribution of different $i j$ fusion channels, i.e., the parton luminosities times $\left|A_{i j}\right|^{2}$. Thus, in principle, quark channels can also be accounted for.

(3) The normalization uncertainty in the $p p$ cross section is controlled by the measured data, which also enters $R_{p A}$.

Significant theoretical uncertainties arise from the $\mu_{F}$ uncertainty in these QCD processes. Different reweighted $n P D F$ sets therefore naturally emerge from the scale variation and we will explain how they should be used in Sec. IV.

We stick to the same $p p$ baseline as in [14] which was slightly extended and improved compared to [76]. It includes the nonprompt $J / \psi$ from $B \rightarrow J / \psi$ data. For the $D^{0}, J / \psi$ and $\Upsilon(1 S)$, a scale variation in the $p p$ baseline itself was performed, besides that in $R_{g}^{\mathrm{Pb}}\left(x, \mu_{F}\right)$.

\section{The PDF reweighting method}

PDF reweighting provides the means for estimating the impact of new experimental data on PDFs without performing a full scale global analysis. Instead, Bayes's theorem is adopted to update the underlying probability distribution and obtain a new updated set of PDFs which takes into account information from the new data. The first use of the reweighting in the context of a PDF analysis was concluded in [97]. Further development of this technique for PDF analyses was carried out resulting in a number of improvements/variants of this method [98-101].

For the purpose of the study in [14] and the current analysis, we used the reweighting procedure outlined in [102] which we briefly summarize here. In the first step, a Hessian PDF set is converted into PDF replicas which have a direct probabilistic interpretation. Since the nPDFs we are using (nCTEQ15 and EPPS16) provide symmetric errors, the conversion allows for an arbitrarily precise reproduction of the corresponding uncertainties ${ }^{7}$ and it can be obtained using the following formula:

\footnotetext{
${ }^{7}$ The precision depends only on the number of used replicas which, in our case, is $10^{4}$.
} 


$$
f_{k}=f_{0}+\sum_{i=1}^{N} \frac{f_{i}^{(+)}-f_{i}^{(-)}}{2} R_{k i},
$$

where $f_{k}$ are $^{8}$ PDF replicas, $f_{0}$ is the central PDF from the Hessian set, $f_{i}^{(+) /(-)}$are the corresponding Hessian error PDFs, $N$ is the number of eigenvector directions for the Hessian set, and $R_{k i}$ are normally-distributed random numbers centered at 0 with a standard deviation of 1 . For such a set of replicas, one can compute the average (corresponding to the Hessian central value) and variance of a PDF-dependent observable $\mathcal{O}$ as

$$
\begin{aligned}
\langle\mathcal{O}\rangle & =\frac{1}{N_{\text {rep }}} \sum_{k=1}^{N_{\text {rep }}} \mathcal{O}\left(f_{k}\right), \\
\delta\langle\mathcal{O}\rangle & =\sqrt{\frac{1}{N_{\text {rep }}} \sum_{k=1}^{N_{\text {rep }}}\left(\mathcal{O}\left(f_{k}\right)-\langle\mathcal{O}\rangle\right)^{2},}
\end{aligned}
$$

where $N_{\text {rep }}$ is the number of replicas.

During the reweighting, each of the PDF replicas is supplemented with a corresponding weight which depends on how well the replica describes the new data. In our analysis, the weight is defined as ${ }^{9}$

$$
w_{k}=\frac{e^{-\frac{1}{2} \chi_{k}^{2} / T}}{\frac{1}{N_{\text {rep }}} \sum_{i}^{N_{\text {rep }}} e^{-\frac{1}{2} \chi_{i}^{2} / T}},
$$

where $T$ is the tolerance criterion used when defining Hessian error PDFs [103] and $\chi_{i}^{2}$ quantifies how well the new data is described. Each nPDF set has been obtained with a specific $T$ value chosen by their authors e.g., $T=35$ for the nCTEQ15 and $T=50$ for EPPS16. In principle, for our reweighting, we could use different values for the tolerance, this, however, would lead to an inconsistent treatment of the data included in the reweighting and the original data used in the nCTEQ15 and EPPS16 nPDF fits [101]. When calculating the $\chi^{2}$ values, the following definition has been used,

$$
\chi^{2}=\left(\frac{1-f_{N}}{\delta^{\text {global }}}\right)^{2}+\Sigma_{i}\left(\frac{T_{i}-f_{N} D_{i}}{\delta_{i}^{\text {uncorr. }}}\right)^{2}
$$

with $\delta^{\text {global }}$ being the global relative error, $T_{i}$ the theoretical prediction, $D_{i}$ the central value of the experimental data, and $\delta_{i}^{\text {uncorr. }}$ the uncorrelated experimental uncertainty. We note that we have accounted for the global (normalization/

\footnotetext{
${ }^{8}$ To be precise, $f$ stands for a vector of functions in the flavor space.

${ }^{9}$ An alternative weight definition can also be used [99]. However, it is more suited to the case of Monte-Carlo-like PDFs [100,101].
}

luminosity) uncertainties by including the factor $f_{N}$ which was fitted for each data set. For completeness, the $\chi^{2}$ values for the considered data sets have been provided in the Appendix A.

After calculating the weights, it is straightforward to compute any PDF-dependent observables as

$$
\begin{aligned}
\langle\mathcal{O}\rangle_{\text {new }} & =\frac{1}{N_{\text {rep }}} \sum_{k=1}^{N_{\text {rep }}} w_{k} \mathcal{O}\left(f_{k}\right), \\
\delta\langle\mathcal{O}\rangle_{\text {new }} & =\sqrt{\frac{1}{N_{\text {rep }}} \sum_{k=1}^{N_{\text {rep }}} w_{k}\left(\mathcal{O}\left(f_{k}\right)-\langle\mathcal{O}\rangle_{\text {new }}\right)^{2} .}
\end{aligned}
$$

More details on the reweighting procedure can be found in [102] and in the other aforementioned references.

\section{Data selection}

In a global PDF fit, HT corrections have to be included in the theory predictions or kinematic cuts have to be imposed such that HT effects are expected to be small in the selected data. For our study, one also needs to select a kinematical region where gluon fusion is the dominant channel and where nPDFs represent the main nuclear effects. This is why we decided in [14] to focus on open- and hidden-HF production in $p A$ collisions at LHC energies. Due to the large Lorentz boost at these energies, as what regards quarkonium production, the heavy-quark pair remains small while traversing the nuclear matter. As such, the breakup of the pair $[104,105]$ is negligible at the LHC whereas it is a potentially large effect at low(er) energies. Along the same lines, we have not considered the more fragile excited states $[\psi(2 S), \Upsilon(2 S), \Upsilon(3 S)]$ and instead we focused on $J / \psi$ and $\Upsilon(1 S)$ data on which the comover effects [59-63] are likely limited. We remind the reader that quarkonium data are, first and foremost, used in our study as a verification that the constraints set by HF LHC data on gluon nPDFs are coherent.

Overall, we have used the following datasets: the ALICE [106] and LHCb [96] $D^{0}$ data; the ALICE [107,108] and LHCb $[109,110] J / \psi$ data; the LHCb [110] $B \rightarrow J / \psi$ data; the ALICE [111], ATLAS [112] and LHCb [113] $\Upsilon(1 S)$ data. The $\chi^{2}$ we obtained and the original values are given as supplemental material to [14]. ${ }^{10}$

Forward $d \mathrm{Au} J / \psi$ RHIC data could have been added. Instead, we preferred to focus on the LHC data at 5 and $8 \mathrm{TeV}$ and to use the RHIC [114,115] and the new LHC $[116,117]$ ones as a cross check as we do here.

\section{RESULTING REWEIGHTED nPDFs}

In [14], we have performed a PDF reweighting using two sets of initial nPDFs: nCTEQ15 and EPPS16, and for each

\footnotetext{
${ }^{10}$ They can be found in Table 2 of https://journals.aps.org/prl/ supplemental/10.1103/PhysRevLett.121.052004/appendix.pdf.
} 
TABLE I. Central scale choice squared, $\mu_{0}^{2}$, for the considered processes. Note that for scale variation we use $\mu_{F}=\xi \mu_{0}$ with $\xi=\{1,2,0.5\}$.

\begin{tabular}{ccccc}
\hline \hline & $J / \psi$ & $D$ & $B \rightarrow J / \psi$ & $\Upsilon(1 S)$ \\
\hline$\mu_{0}^{2}$ & $M_{J / \psi}^{2}+P_{T, J / \psi}^{2}$ & $4 M_{D}^{2}+P_{T, D}^{2}$ & $4 M_{B}^{2}+\frac{M_{B}^{2}}{M_{J / \psi}^{2}} P_{T, J / \psi}^{2}$ & $M_{\Upsilon}^{2}+P_{T, \Upsilon}^{2}$ \\
\hline \hline
\end{tabular}

of these nPDFs we have used data for $J / \psi, D$-meson, $B \rightarrow$ $J / \psi$ and $\Upsilon(1 S)$ production from $p \mathrm{~Pb}$ collisions at the LHC to produce four reweighted nPDF (RnPDF) sets. The reweighting for each data set was done independently and three sets were obtained from a scale variation using the following scale choices: $\mu=\left\{\mu_{0}, 2 \mu_{0}, 0.5 \mu_{0}\right\}$ (see Table I for the values of $\mu_{0}$ ).

Going further than in [14], we have created, for the current study, an additional RnPDF set for each set of data by combining the uncertainties obtained from the scale variations. The combined set is produced by simply taking all replicas from reweightings with different scale choices and treating them as equally probable.

This gives us $3+1$ RnPDF sets for each combination of initial nPDF sets (nCTEQ15 or EPPS16) and each data type which together gives 32 new RnPDF sets. Since we want to provide all these new RnPDFs to the public such that they can be used in other studies, we have converted them into corresponding Hessian sets which are handier to use than the (many) PDF replicas which were initially obtained. In order to produce Hessian sets out of PDF replicas we use the mc2hessian program $[118,119]$. For the reweighted Hessian sets we use the same number of error sets as in the original nPDFs which in the case of nCTEQ15 is 32 and in the case of EPPS16 is 40. One should however note that the obtained errors are symmetric and as a result it is sufficient to provide correspondingly only 16 and 20 error PDFs. The RnPDF errors for the resulting sets should be computed using the following prescription

$$
\Delta \mathcal{O}=\sqrt{\sum_{k}\left(\mathcal{O}\left(f_{k}^{+}\right)-\mathcal{O}\left(f_{0}\right)\right)^{2}},
$$

where $\mathcal{O}$ is a PDF-dependent observable and $k$ goes over 16 or 20 error PDFs. ${ }^{11}$

The comparison of the reweighted and original gluon distributions are presented in Fig. 2. We should highlight here that the plots (and the corresponding LHAPDF files) features PDF uncertainties at 90\% Confidence Level (CL). This is in accordance with the original nPDF sets we used (nCTEQ15 and EPPS16) but it differs from what we

\footnotetext{
${ }^{11}$ The original nCTEQ15 and EPPS16 LHAPDF sets provided both "plus" and "minus" error PDFs and as a result the appropriate formula for calculating PDF uncertaintiy was $\Delta \mathcal{O}=$ $\frac{1}{2} \sqrt{\sum_{k}\left(\mathcal{O}\left(f_{k}^{+}\right)-\mathcal{O}\left(f_{k}^{-}\right)\right)^{2}}$ instead.
}

presented in [14] where we had used $68 \%$ CL. $^{12}$ Additional plots showing a detailed comparison of gluon nuclear modifications for different reweightings both at 68\% CL and 90\% CL are presented in the Appendix B.

In order to confront the resulting distributions with recent nPDFs, in Fig. 3, we compare the gluon NMFs obtained in the HF reweightings with the results from the nCTEQ15WZ [28] and nNNPDF20 [11] nPDF sets. None of these two nPDFs used the LHC HF data we employed in our analysis in the fits and, as such, they do not have stringent constraints on the gluon distribution especially in the low- $x$ region (since most of the constraints in these sets are coming from the LHC $W / Z$ boson data which have a kinematic reach to around $x \gtrsim 10^{-3}$ ). Nevertheless, it is interesting to see the comparison which clear shows that the HF data is crucial for pinning down the low- $x$ gluon distribution, confirming that the gluon is shadowed at small values of $x .^{13}$

We note that in Fig. 3 we show a single error band for each of the HF RnPDFs. This error band is an envelope of error bands originating from the reweightings performed varying the factorization scale (see Fig. 2 and Table I).

Furthermore, since the atomic mass, $A$, of lead and gold nuclei are very close (208 vs 197) nuclear modifications for these two nuclei are also very similar. We used this fact and assumed that the results of reweighting using the $p \mathrm{~Pb}$ LHC HF data can be directly transferred to the case of gold. To do so, we simply applied the weights obtained for $\mathrm{Pb} \mathrm{nPDF}$ replicas to the corresponding Au nPDF replicas. This amounts to assuming that the $A$ dependence in our RnPDFs is the same as that of the original nPDFs. That way, one can see the impact of HF $p \mathrm{~Pb}$ LHC data on $\mathrm{Au}$ nPDFs and confront such new information with $p / d \mathrm{Au}$ data from RHIC.

To allow others to use these results we provide LHAPDF grid files for the obtained Hessian sets. We supply the sets for lead and gold nuclei originating from nCTEQ15 and EPPS16 $J / \psi, D$, and $B \rightarrow J / \psi$ meson data. We refrain from providing also the sets obtained from reweightings using $\Upsilon(1 S)$ data as the impact of these data on the original nPDFs was marginal. The LHAPDF grid files will be available at http://nloaccess.in2p3.fr/HF-LHC-RW-2017.

\footnotetext{
${ }^{12}$ For nCTEQ15, the reweighting itself was performed at $68 \%$ CL but for the convenience of the users we have converted the resulting nPDFs to the $90 \% \mathrm{CL}$ which is the standard used in the community. For EPPS16, the reweighting was performed at 90\% CL.

${ }^{13}$ One should note that the relatively small uncertainty of the nCTWQ15WZ gluon distribution at $x \sim 10^{-4}$ (thus below the kinematics of the data) and EPPS16 gluon at $x \sim 10^{-5}$ is partially an artifact of the used parametrizations. The actual gluon uncertainty at small $x$ is larger and closer to the uncertainty provided by the nNNPDF20 distribution. This in fact means that the information provided by the HF data, which we discuss here, is even more relevant.
} 


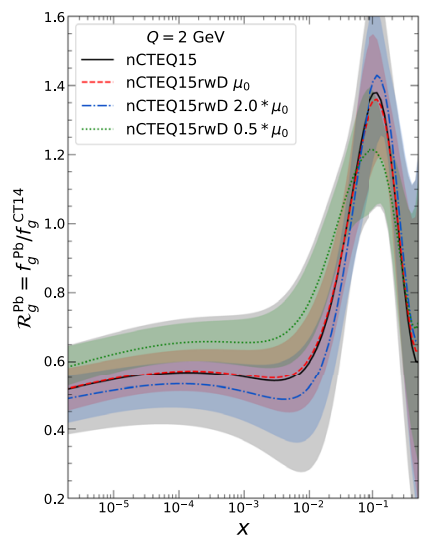

(a)

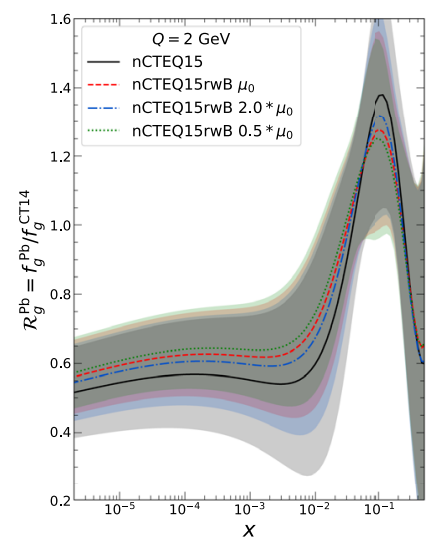

(c)

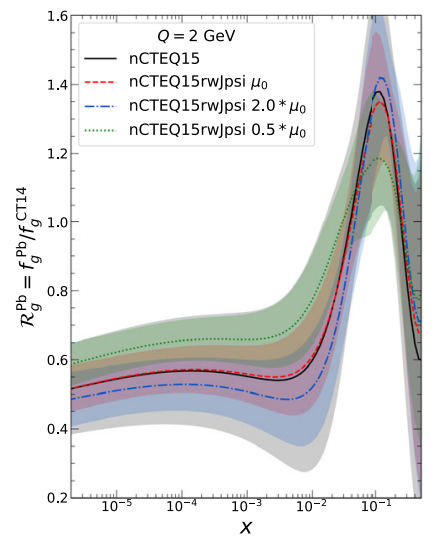

(e)

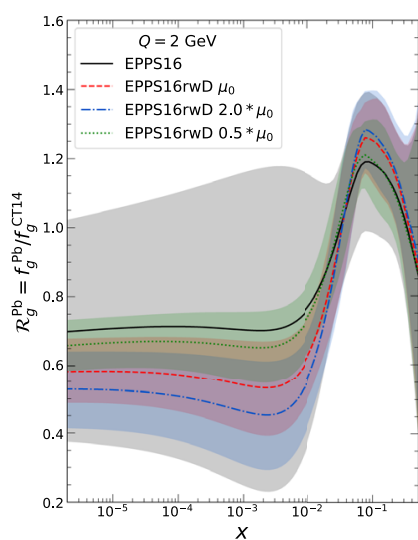

(b)

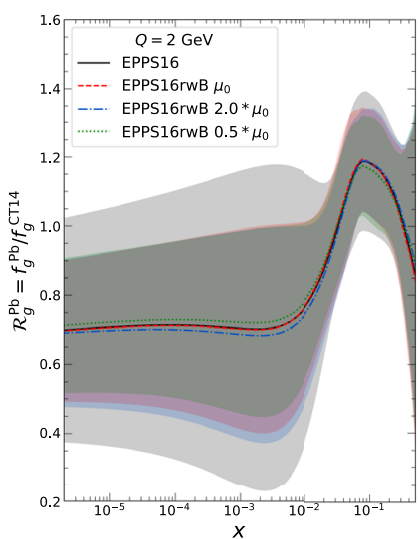

(d)

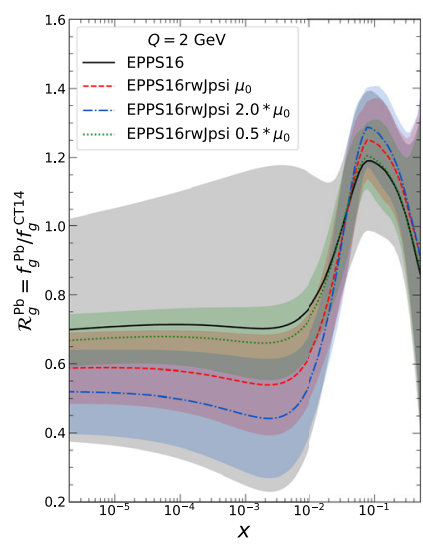

(f)

FIG. 2. Comparison of the (un)reweighted gluon nuclear ratio for Pb from the nCTEQ15 (left) and EPPS16 (right) nPDFs for reweightings with (from top to bottom): $D$ meson, $B \rightarrow J / \psi$, and $J / \psi$ data. The scale variation was performed and shown on each plot about $\mu_{0}$ in Table I. (a) $D$-reweighted nCTEQ15. (b) $D$-reweighted EPPS16. (c) $B \rightarrow J / \psi$-reweighted nCTEQ15. (d) $B \rightarrow J / \psi$-reweighted EPPS16. (e) $J / \psi$-reweighted nCTEQ15. (f) $J / \psi$-reweighted EPPS16.

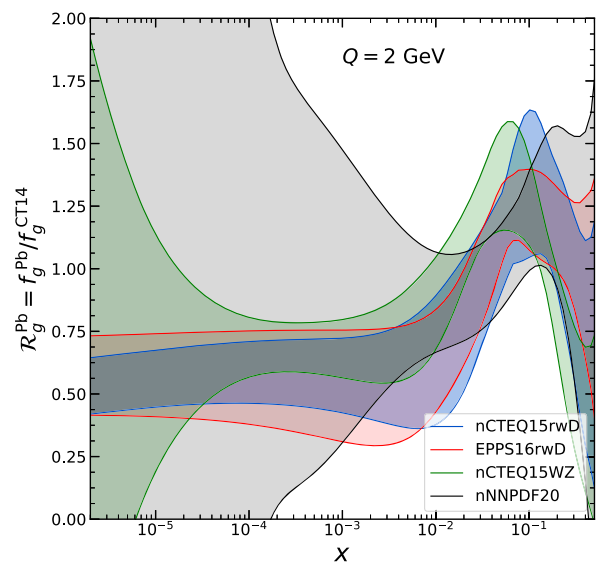

(a)

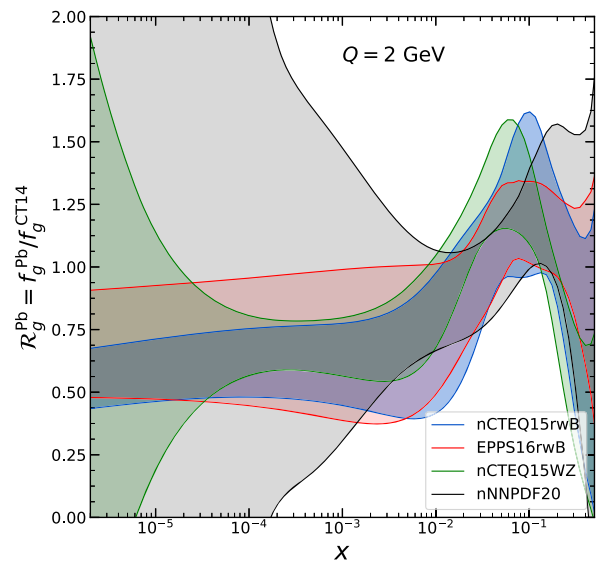

(b)

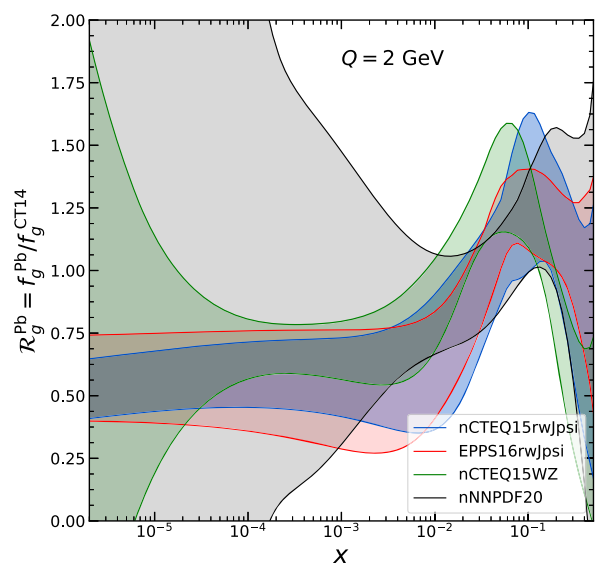

(c)

FIG. 3. Comparison of the reweighted gluon nuclear ratio for $\mathrm{Pb}$ from the nCTEQ15 and EPPS16 with nCTEQ15WZ [28] and nNNPDF20 [11]. The ratio is taken with respect to the CT14nlo proton PDF [120]. The uncertainties for the RnPDFs are computed as envelopes of uncertainties for sets obtained with different scales. (a) $D$ RnPDFs. (b) $B \rightarrow J / \psi$ RnPDFs. (c) $J / \psi$ RnPDFs. 


\section{RESULTS}

As we explained above, a data selection is necessary to minimize HT effects [30]. In addition, we recall that our parametrization of the hard scattering neglects the (anti-) quark contributions. These are irrelevant at the LHC. However, it is important that one remains in a kinematical region where gluon fusion dominates to apply this parametrization. For charmonia, this remains the case down to the energy range of RHIC [121-123] and the LHC in the fixed-target mode [124-127] but it is also important to keep away from those regions where other nuclear effects than those which can be encapsulated in the nPDFs are believed to be important. This explains why we originally restricted our reweighting study to $\mathrm{HF}$ production in $p A$ collisions at LHC energies.

We could also have added the forward $d \mathrm{Au} J / \psi$ RHIC data. Instead, we preferred to focus on the LHC data and to use the RHIC [114,115] and recent LHC [116,117] ones as cross checks. We also note that adding the RHIC data would in fact have constrained Au nPDFs. Here, what we rather do is to assume that the relative $A$ dependence of $\mathrm{Au}$ and $\mathrm{Pb}$ shadowing is the same as in the original nPDFs.

We extend this validation by also showing comparisons with a number of data sets which appeared after our study $[128,129]$ and which can be considered as predictions since the nPDFs were left unchanged. However, our objective is not to be exhaustive but rather to illustrate that our RnPDFs provide a first good estimate of nuclear effects at work on the production of the corresponding particles and that our released LHAPDF grids can be used as such. Our examples have also been chosen to explain which LHAPDF grids to use and how, that is with which $\mu_{F}$ scale choices.

We should indeed distinguish two cases. The first corresponds to the situation where a LHAPDF grid has been reweighted on the data of same process (or a very similar one) as that for which one wishes to provide NMF predictions. In such a case, the $\mu_{F}$ uncertainties are likely highly correlated even if the $x$ range is not similar. ${ }^{14}$ As such, we advocate that the NMF should be computed for three scale choices $[\xi=(0.5,1.0,2.0)]$ by using the RnPDF sets corresponding to the same three scale values used in the reweighting and then to take the envelope of the resulting NMF uncertainties. This is what we will show next for $J / \psi$ and charm production.

On the contrary, if the process for which one wishes to compute the NMF is a different one, e.g., di-jet vs charm production, we find it more reasonable to consider that the

\footnotetext{
${ }^{14}$ We however note that, for low- $P_{T}$ quarkonia at NLO, the NLO QCD corrections generate a subtle $\mu_{F}$ vs $x$ interplay which results in negative cross sections at increasing energies for $\mu_{F}$ larger than the quarkonium mass. In such a case, we recently advocated [130] the use of a specific $\mu_{F}$ scale choice. So far, this has only been addressed for $\eta_{Q}$ production. Once the $J / \psi$ case is addressed, reweighting of nPDFs with this scale choice would be performed.
}

$\mu_{F}$ uncertainties are not correlated. In such a case, one should rather use the constraints from the reweighting without any prior and take all the eigensets obtained with the different scale choices on the same footage. Hence, we advocate the use of the new merged grid, the computation of the NMF for three scale choices [still $\xi=(0.5,1.0,2.0)$ ] and the consideration of the envelope of the resulting uncertainties. Since we did not provide LHAPDF grid files for the reweighting with the $\Upsilon$ data, the prediction for the $\Upsilon$ NMFs which we show next are obtained likewise, using the gluon nPDF reweighted with charm data. We have also done so for $B$ predictions. We could have used those from beauty or $J / \psi$ in both cases as well.

\section{A. $J / \psi$ data at RHIC at $\sqrt{s_{\mathrm{NN}}}=200 \mathrm{GeV}$}

Let us start with some comparisons with RHIC data at $\sqrt{s}=200 \mathrm{GeV}$ both for $d \mathrm{Au}^{15}$ and $p$ Au collisions as a function of $y_{\text {c.m.s. }}$ since they illustrate that there is indeed an universality in the suppression of $J / \psi$ forward data at high energies which can be reproduced by nPDF effects only.

Indeed, one can see in Fig. 4 and Fig. 5 that the magnitude of the suppression in the forward region is well accounted for by the nPDFs reweighted on the $J / \psi$ LHC data. We recall that the shown uncertainty results from the envelope of the bands found by taking the three $J / \psi$ RnPDFs using three scales evaluated at the same three scale choices. As we just explained, this amounts to assuming that the factorization scale in the hard scattering is fully correlated between the LHC and RHIC energies. Nevertheless, it remains unknown and should be varied.

On the other hand, one clearly sees that the backward data cannot be described, and to a lesser extent the centralrapidity ones. This is absolutely not surprising - and it is well known that the same happens with the original unreweighted nPDFs [76] - since, at these energies and rapidities, the $J / \psi$ has the time to fully form while traversing the nucleus. As such, additional (final-state) effects need to be considered. For instance, they can be encapsulated in an effective absorption. We guide the interested reader to a series of works treating these effects $[70,104,131,132]$. In what follows, we therefore naturally do not discuss further the $P_{T}$ dependence of the NMFs in this rapidity region at RHIC. Figure 6 and Fig. 7 respectively show the corresponding $R_{d \mathrm{Au}}$ and $R_{p \mathrm{Au}}$ vs $P_{T}$ which are found to be fairly well reproduced by our RnPDFs.

\section{B. $D^{0}$ at the $\mathrm{LHC} \sqrt{s_{\mathrm{NN}}}=5.02 \mathrm{TeV}$}

We start our list of comparisons with LHC data with the $D$-meson ones which are representative of charm data. At this stage, since our examples are mainly illustrative, we

\footnotetext{
${ }^{15}$ Our results for $d \mathrm{Au}$ collisions are obtained by neglecting any nuclear effect in the deuteron.
} 


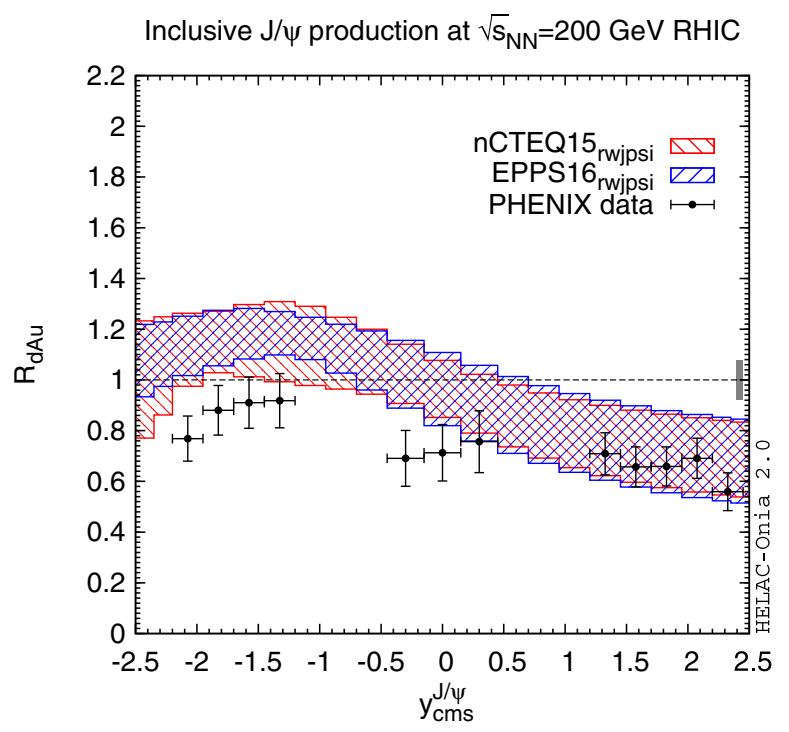

FIG. 4. $J / \psi R_{d \mathrm{Au}}$ as a function of $y_{\text {c.m.s. }}$ at $\sqrt{s_{\mathrm{NN}}}=200 \mathrm{GeV}$ computed using our $J / \psi$-RnPDFs compared to PHENIX data [114].

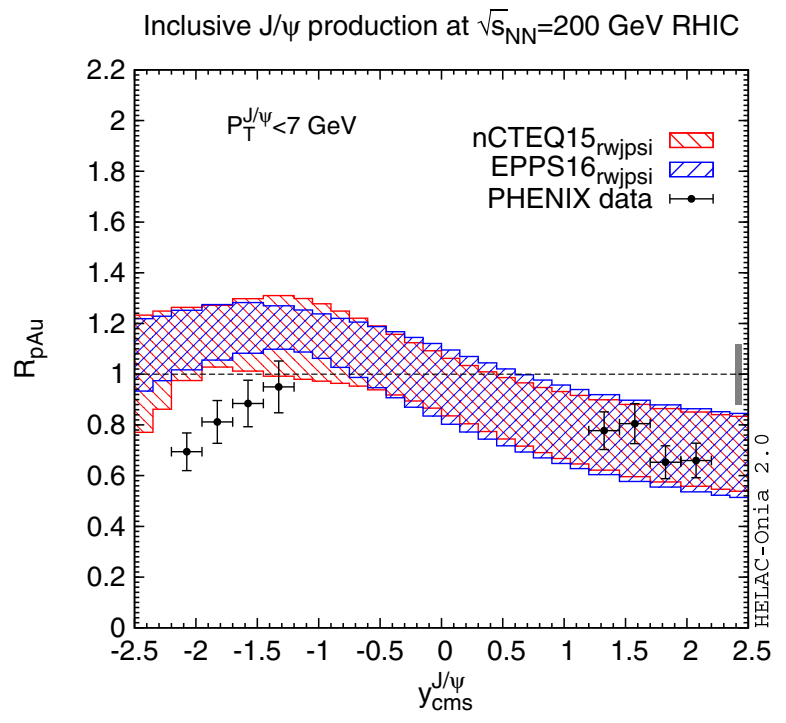

FIG. 5. $J / \psi R_{p \mathrm{Au}}$ as a function of $y_{\text {c.m.s. }}$ at $\sqrt{s_{\mathrm{NN}}}=200 \mathrm{GeV}$ computed using our $J / \psi$-RnPDFs compared to PHENIX data [128].

have decided not to consider lepton-from-charm data, which are in any case much more complex theory wise to compute. We recall that our objective here is not to demonstrate that one can account for the entire set of existing data but to illustrate how and where to use our RnPDF sets and what conclusion to draw from NMFs which would then be obtained.

Our first example is in fact a consistency check for which we show the NMF for $D^{0}$ at $\sqrt{s_{\mathrm{NN}}}=5.02 \mathrm{TeV}$ obtained from the $D$-RnPDFs precisely using $5.02 \mathrm{TeV}$ data. These have been computed while taking into account the scale
Inclusive $\mathrm{J} / \psi$ production at $\sqrt{\mathrm{s}}_{\mathrm{NN}}=200 \mathrm{GeV} \mathrm{RHIC}$

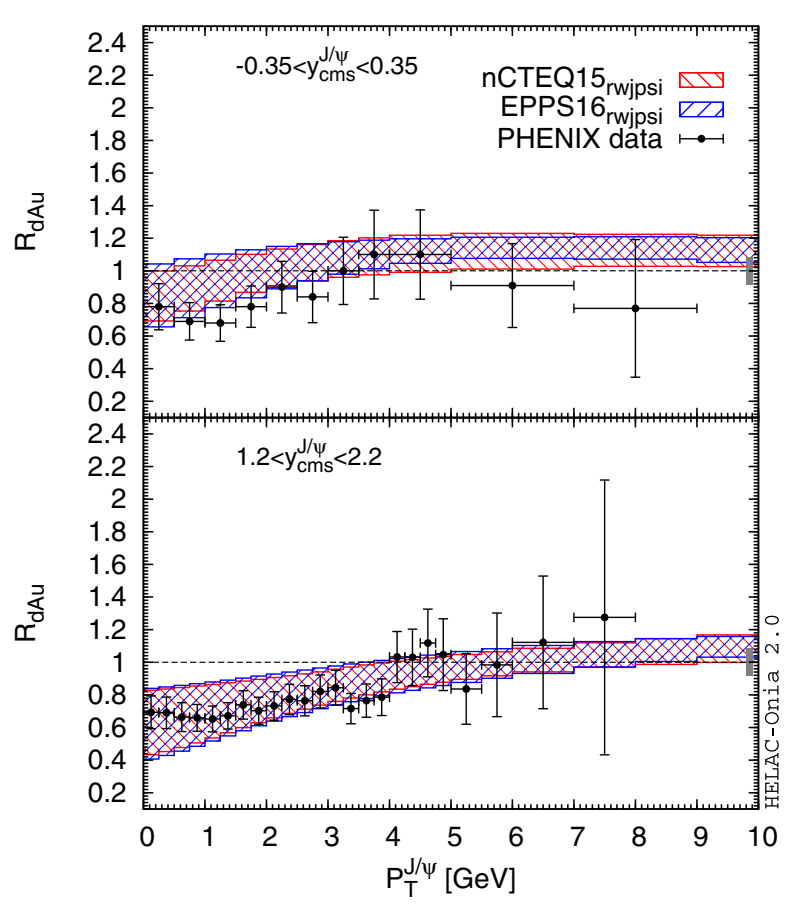

FIG. 6. Computed $J / \psi R_{d \mathrm{Au}}$ as a function of $y_{\text {c.m.s. }}$ at $\sqrt{s_{\mathrm{NN}}}=$ $200 \mathrm{GeV}$ using our $J / \psi$-RnPDFs compared to PHENIX data [115].

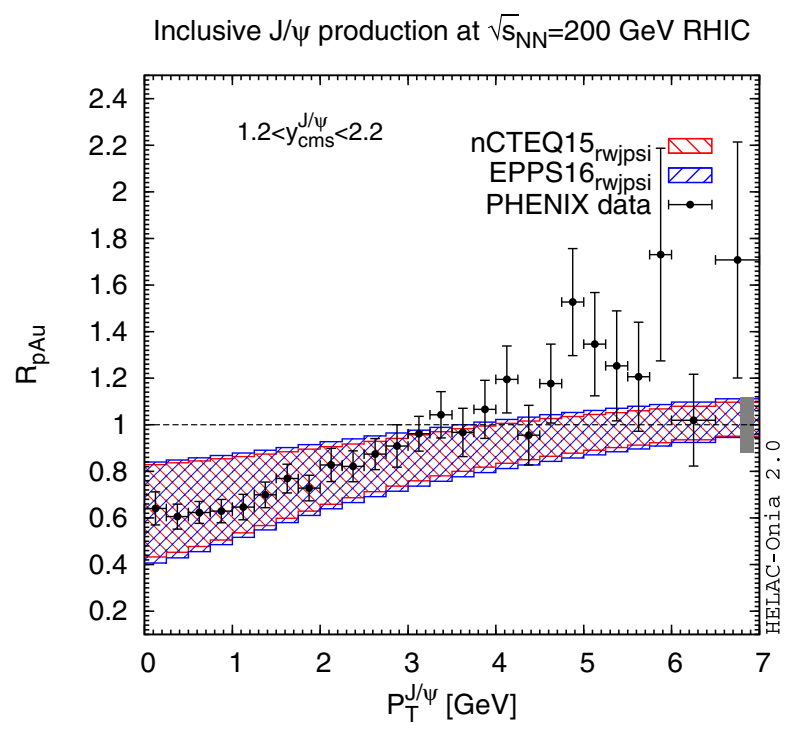

FIG. 7. Computed $J / \psi R_{p \text { Au }}$ as a function of $y_{\text {c.m.s. }}$ at $\sqrt{s_{\mathrm{NN}}}=$ $200 \mathrm{GeV}$ using our $J / \psi$-RnPDFs compared to PHENIX data [128].

correlation as explained above. Figure 8 shows the same agreement with the $R_{p \mathrm{~Pb}}$ as a function of $y_{\text {c.m.s. }}$ measured by LHCb as that obtained in Ref. [14]. The only difference indeed lies in the procedure to derive the uncertainties with the scale correlation. A similar correspondence is found for the $P_{T}$ dependence (see Fig. 9). 


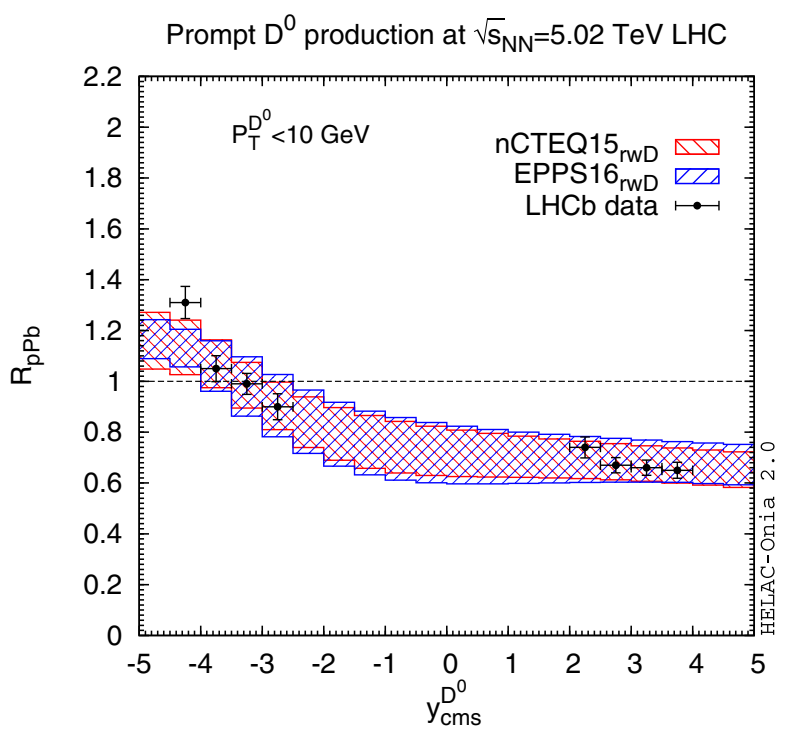

FIG. 8. Computed $D^{0} R_{p \mathrm{~Pb}}$ as a function of $y_{\text {c.m.s. }}$ at $\sqrt{s_{\mathrm{NN}}}=$ 5.02 TeV using our $D$-RnPDFs compared to LHCb data [96].

Regarding other $D^{0}$ data, as of now, we are only able to compare with the central-rapidity data from ALICE as shown in Fig. 10. These admittedly exhibit much larger experimental uncertainties and only hint at a possible smaller suppression than what one would expect from our RnPDFs. More precise data at $8.16 \mathrm{TeV}$ for instance at backward and forward rapidities from $\mathrm{LHCb}$ would be

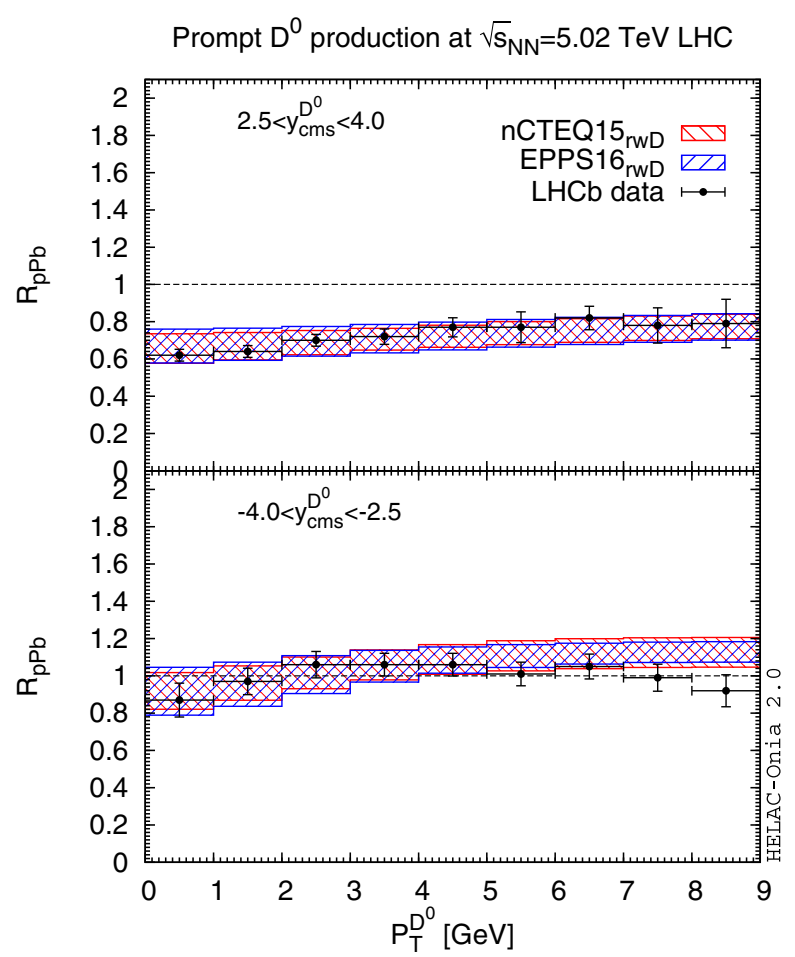

FIG. 9. $D^{0} R_{p \mathrm{~Pb}}$ as a function of $P_{T}$ at $\sqrt{s_{\mathrm{NN}}}=5.02 \mathrm{TeV}$ at forward (top) and backward (bottom) $y_{\text {c.m.s. }}$ computed using our $D$-RnPDFs compared to $\mathrm{LHCb}$ data [96].

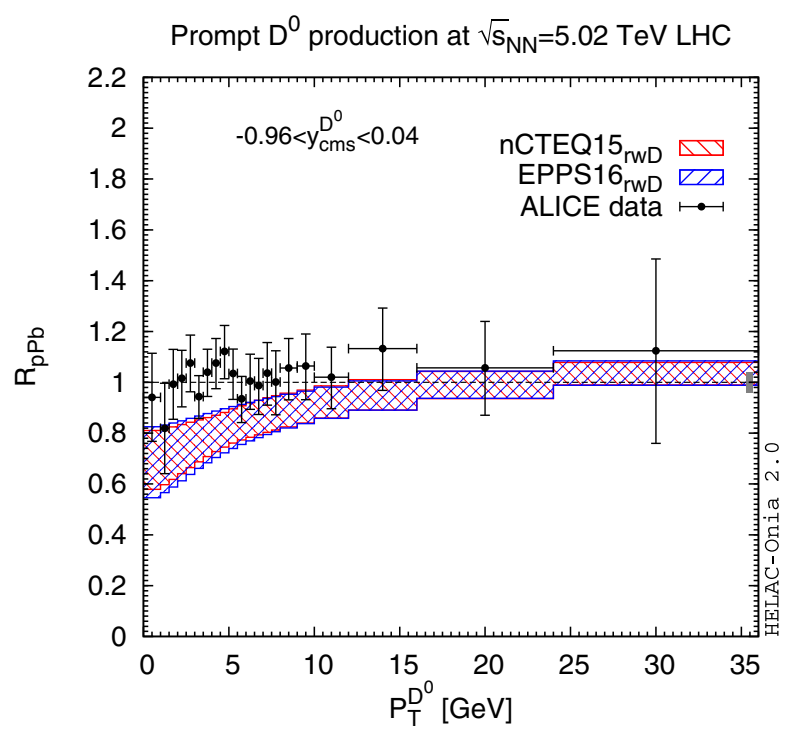

FIG. 10. $D R_{p \mathrm{~Pb}}$ as a function of $P_{T}$ at $\sqrt{s_{\mathrm{NN}}}=5.02 \mathrm{TeV}$ computed using our $D$-RnPDFs compared to ALICE data [134].

welcome, along the lines of their preliminary analysis of $R_{\mathrm{FB}}[133]$.

\section{C. $J / \psi$ at $\sqrt{s_{\mathrm{NN}}}=8.16 \mathrm{TeV}$}

We now move on to the $J / \psi$ case. Since we have $J / \psi$ RnPDFs, we have used them while taking into account the scale correlation. In this case, we only show comparisons with ALICE data at $8.16 \mathrm{TeV}$ which we did not include in our initial reweighting analysis [14]. Figure 11 showswithout much surprise as well- that the magnitude of the NMF is well reproduced by our $J / \psi$-reweighted PDFs.

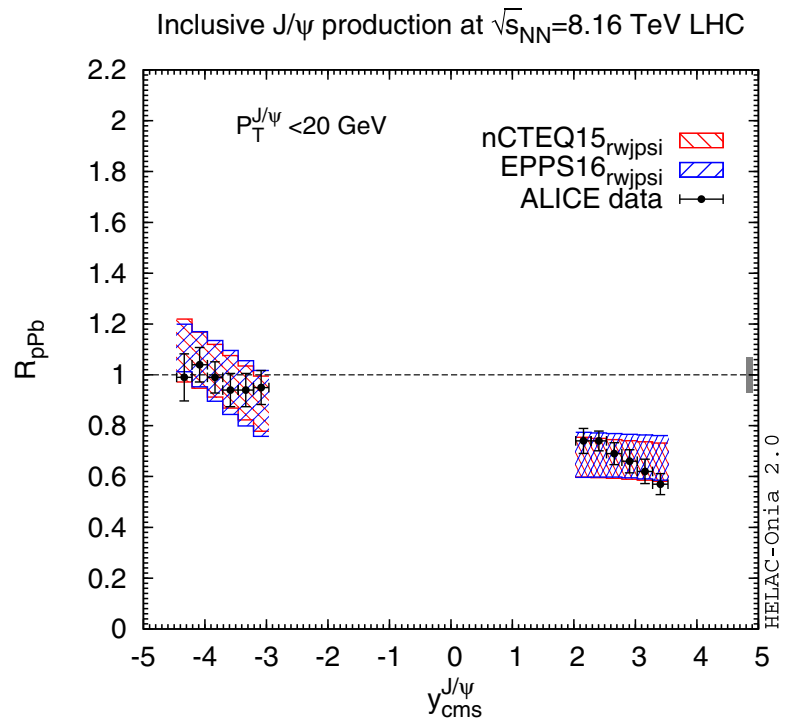

FIG. 11. $J / \psi R_{p \mathrm{~Pb}}$ as a function of $y_{\text {c.m.s. }}$ at $\sqrt{s_{\mathrm{NN}}}=8.16 \mathrm{TeV}$ computed using our $J / \psi$-RnPDFs compared to ALICE data [129]. 
Inclusive $\mathrm{J} / \psi$ production at $\sqrt{\mathrm{N}}_{\mathrm{NN}}=8.16 \mathrm{TeV}$ LHC

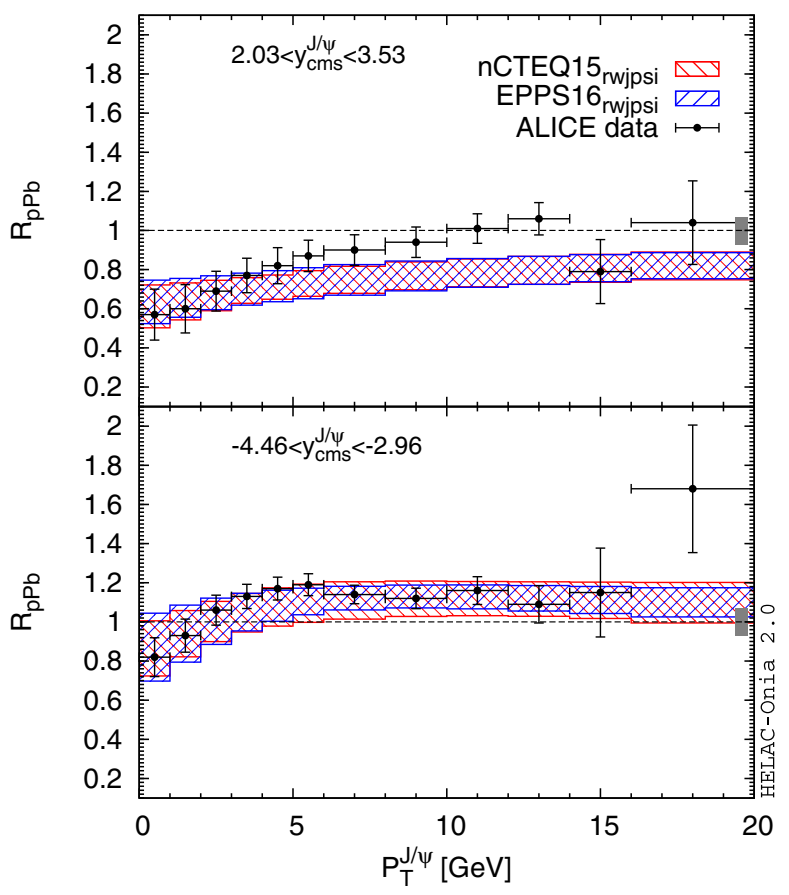

FIG. 12. $J / \psi R_{p \mathrm{~Pb}}$ as a function of $P_{T}$ at $\sqrt{s_{\mathrm{NN}}}=8.16 \mathrm{TeV}$ at forward (top) and backward (bottom) $y_{\text {c.m.s. }}$ computed using our $J / \psi$-RnPDFs compared to ALICE data [129].

As for the $P_{T}$ dependence shown on Fig. 12, it is particularly well accounted for in the backward region, less in the forward region of $P_{T}$. One could be tempted to attribute this to the growing impact of nonprompt $J / \psi$ for increasing $P_{T}$. Indeed, around $P_{T}=10 \mathrm{GeV}$, this nonprompt fraction has already tripled compared to the $P_{T^{-}}$ integrated value to reach $30 \%$. However, anticipating our $B$ results, it is not obvious that this is the case. Another possible explanation is that the agreement in the backward region is coincidental and comes from the onset of the absorption. In such a case, it could be that, in general, the larger $P_{T}$ region is not well accounted for by our RnPDFs. Once again, we wish to keep this discussion rather descriptive as final physical conclusions would require a full $\mathrm{nPDF}$ fit to see if these data are or not reproducible by LT nPDF effects alone.

\section{D. $B$ at $\sqrt{s_{N N}}=8.16 \mathrm{TeV}$}

We now come to the $B$ meson case. Because the $B$ data were not precise enough when we performed our reweighting analysis [14], the results we obtained only showed marginal differences with the original nPDFs. As such, we find it to be a neat example to illustrate how to use our RnPDFs to compute NMFs for processes which are not connected to those used in the reweighting. In this case, we have used our $D$-RnPDFs with the combined three-scale results to evaluate the NMFs for three scales and have taken the resulting envelope.
Inclusive $\mathrm{B}^{+}$production at $\sqrt{\mathrm{S}}_{\mathrm{NN}}=8.16 \mathrm{TeV} \mathrm{LHC}$

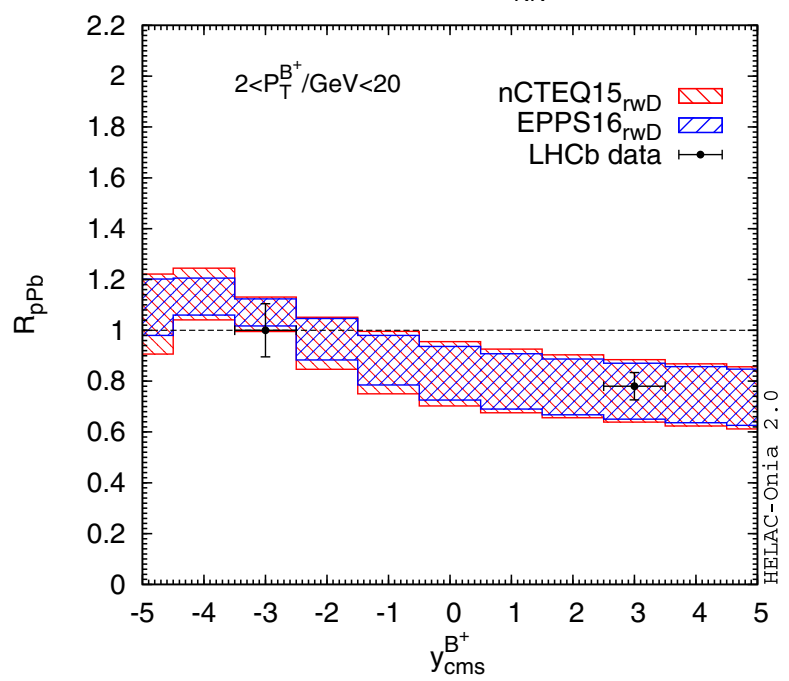

FIG. 13. $B^{+} R_{p \mathrm{~Pb}}$ as a function of $y_{\text {c.m.s. }}$ at $\sqrt{s_{\mathrm{NN}}}=8.16 \mathrm{TeV}$ computed using our $D$-RnPDFs compared to LHCb data [135].

Figure 13 shows the resulting $y_{\text {c.m.s. }}$ dependence which agrees very well with both $\mathrm{LHCb} B^{+}$data points at 8.16 TeV. We pushed the comparison further with the $P_{T}$ dependence shown in Fig. 14 which is in good agreement within the experimental and theoretical uncertainties. We note that we could have performed more such comparisons, with $D$-RnPDF, in particular with $J / \psi$ from $B$ data

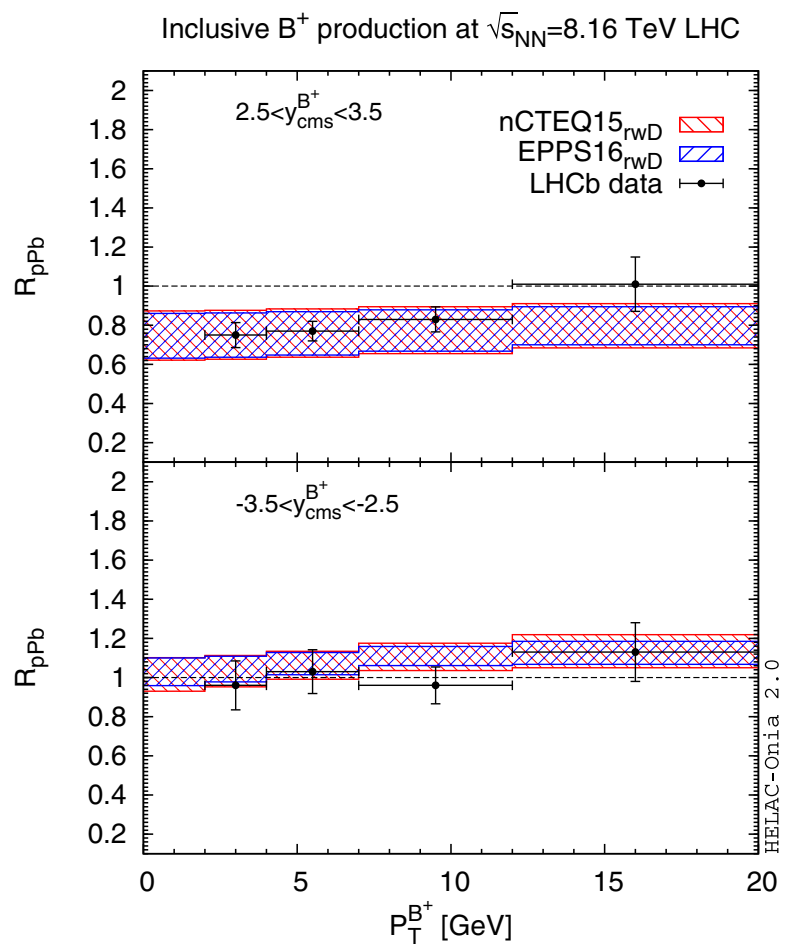

FIG. 14. $B^{+} R_{p \mathrm{~Pb}}$ as a function of $P_{T}$ at $\sqrt{s_{\mathrm{NN}}}=8.16 \mathrm{TeV}$ at forward (top) and backward (bottom) $y_{\text {c.m.s. }}$ computed using our $D$-RnPDFs compared to LHCb data [135]. 
Inclusive $\Upsilon(1 \mathrm{~S})$ production at $\sqrt{\mathrm{S}}_{\mathrm{NN}}=8.16 \mathrm{TeV} \mathrm{LHC}$

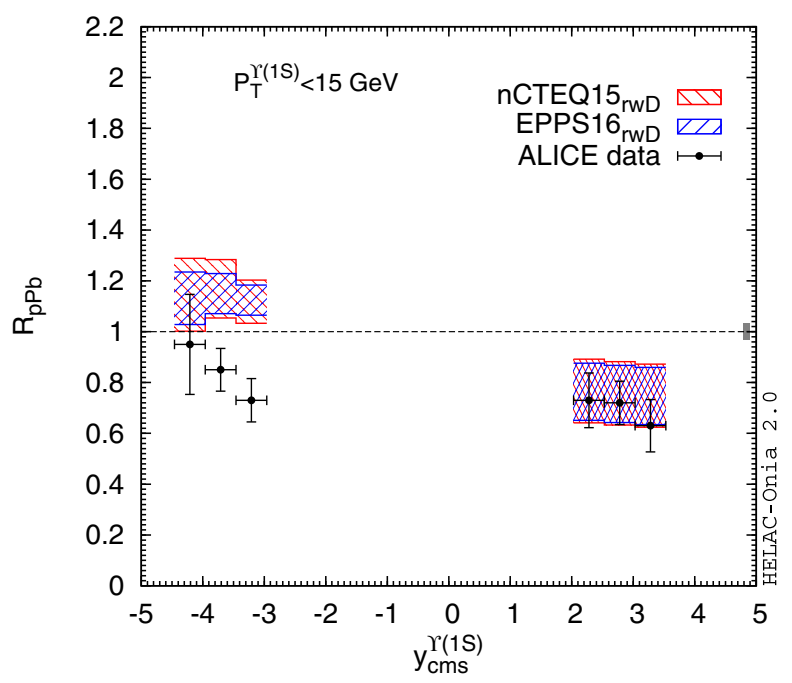

FIG. 15. $\Upsilon(1 S) R_{p \mathrm{~Pb}}$ as a function of $y_{\text {c.m.s. }}$ at $\sqrt{s_{\mathrm{NN}}}=$ $8.16 \mathrm{TeV}$ computed using our $D$-RnPDFs compared to ALICE data [136].

from LHCb. However, we recall that our objective is certainly not to be exhaustive but to illustrate the usage of our reweigthed nPDFs.

\section{E. $\Upsilon(1 S)$ at $\sqrt{s_{N N}}=8.16 \mathrm{TeV}$}

Our last set of comparisons concerns $\Upsilon(1 S)$ data. Like for $B$ mesons, we have used $D$-RnPDFs. Figure 15 shows the resulting $y_{\text {c.m.s. }}$ dependence. We find a good agreement in the forward region, but not in the backward region. It seems that the peak generated by the antishadowing is simply absent. Either the $D$ data tend to make it too strong, ${ }^{16}$ or the $\Upsilon(1 S)$ data are suppressed by another mechanism. ${ }^{17}$ The NMF $P_{T}$ dependence, shown in Fig. 16, confirms this observation.

\section{F. Note on the di-jet data}

After our initial reweighting study came out, the CMS collaboration claimed the first observation [137] of a depletion of gluons in $\mathrm{Pb}$ at large $x$ based on an analysis of the di-jet yield ratio in $p \mathrm{~Pb}$ and $p p$ collisions as a function of the di-jet pseudorapidity, $\eta_{12}$. Such a suppression would correspond to a gluon EMC suppression which was already hinted at by PHENIX $\Upsilon$ data [71].

It would be very insightful to do a comparison for such ratio using our HF RnPDFs to see if the data constraints concur to the same effect. Yet, we would like to stress that the $p p$ data are not well accounted for by the fixed-order

\footnotetext{
${ }^{16}$ We indeed note here that the most backward point for $D$ on Fig. 8 is quite high and may have driven the antishadowing in our $D$ RnPDFs too high.

${ }^{17}$ For instance, comovers could induce a shift down of $R_{p \mathrm{~Pb}}$ by 0.05 [60].
}

Inclusive $\Upsilon(1 \mathrm{~S})$ production at $\sqrt{\mathrm{S}}_{\mathrm{NN}}=8.16 \mathrm{TeV} \mathrm{LHC}$

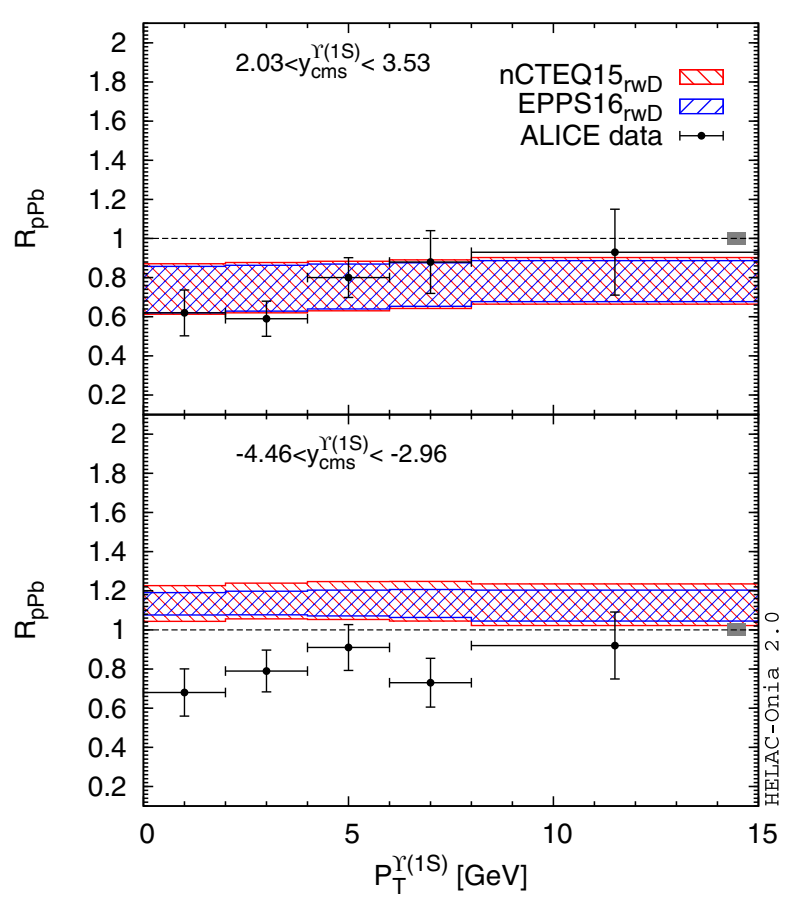

FIG. 16. $\Upsilon(1 S) R_{p \mathrm{~Pb}}$ as a function of $P_{T}$ at $\sqrt{s_{\mathrm{NN}}}=8.16 \mathrm{TeV}$ at forward (top) and backward (bottom) $y_{\text {c.m.s. }}$ computed using our $D$ RnPDFs compared to ALICE data [136].

NLO computations and large (positive and negative) $\eta_{12}$. We refer to a neat discussion by Eskola et al. [138] for more details and a discussion that the deviation may be accounted by modifying the proton PDFs. The discrepancies can easily be on the order of the expected size of the nuclear effects. This happens in the kinematical regions where one would need to look for such a depletion at large $x$ or shadowing at low $x$.

If we were to bypass a fixed-order analysis by using the same method we have proposed in [76] by parametrizing the amplitude squared, $|A|^{2}$, without enough kinematical lever arm in its determination, we would probably hide such a disagreement in $|A|^{2}$ and our predictions could well be wrong. Yet, in principle, what matters most in our nPDFbased NMF predictions is the relevant $x_{1}-x_{2}-Q$ region probed by the scattering. We agree that it is somewhat unlikely that the observed disagreement could be the signal of a phenomenon significantly altering the kinematic of the scattering; this is however not completely excluded if this comes from kinematically enhanced NNLO QCD corrections as it appears that the di-jet data are better described at NNLO [139]. Yet, until this issue is settled, we prefer to refrain from performing NMF predictions for di-jets. We in fact leave this to the interested reader to do so since our RnPDFs are now usable by anyone thanks to our released LHAPDF grids. We however suggest a careful reading of $[138,139]$. 


\section{CONCLUSIONS}

We have presented a follow-up of our reweighting study [14] of two of the recent global fits of nuclear parton densities at NLO (nCTEQ15 [22] and EPPS16 [9]) which consists in a release of the corresponding LHAPDF grids with Hessian uncertainties. These will allow anyone to employ the constraints encoded in the HF experimental data set which we have used for the reweighting in order to perform computations of observables like cross sections or NMFs.

We had indeed focused on HF LHC data whose predicted yields are however very sensitive to the factorization scale, $\mu_{F}$. Since the magnitude of the nuclear effects is also strongly $\mu_{F}$ sensitive, this resulted in a significant dependence of the reweighting on the $\mu_{F}$ we choose. For instance, if one takes a value smaller than the default value, say the transverse mass of the produced particle, the resulting shadowing in the RnPDFs will be weaker. On the contrary, if one reweights with a larger $\mu_{F}$, the resulting RnPDFs will always exhibit a stronger shadowing compared to the former case.

This naturally induced a significant uncertainty, which we had found [14] to be as large as that of the resulting $D$ and $J / \psi$-RnPDFs themselves. As we noted, this is less problematic for the bottom-quark sector but the data are not yet precise enough to yield valuable constraints. We have thus generated several RnPDF grids to be used with correlated $\mu_{F}$ choices if one performs predictions for similar systems as that used for the reweighting. In addition, we release here new RnPDFs with combined scale uncertainty to be used when one assumes no correlation between these scales, e.g., to predict isolated photon or $\Upsilon$ NMFs from $D$-RnPDFs.

We have thus found it useful to show a selection of comparisons with experimental data to illustrate how to use our RnPDF grids and what to expect from them. In most of the cases, we find a very good agreement with LHC data. It is of course expected in the case of the $8 \mathrm{TeV} \mathrm{J} / \psi$ data since one set by $\mathrm{LHCb}$ was already included in our reweighting. For the other $8 \mathrm{TeV}$ data, the agreement indicates that the $x$ dependence of the RnPDFs correctly captures the energy dependence of the $R_{p \mathrm{~Pb}}$ and, to some extent as well, highlights the coherence between different LHC data sets. In the case of $200 \mathrm{GeV}$ RHIC data, the agreement we have obtained is even more striking and indeed shows the $x$ dependence of the RnPDFs provide a good description of the gluon distribution up to the upper end of the shadowing region.

Before a full NLO fit using these data is performed, we believe that our RnPDFs can safely be used when the conventional nPDFs show too large uncertainties preventing any physics conclusions. Yet, one should keep in mind that if a strong disagreement is found, it will always be necessary to wonder if a new fitting procedure, which by construction will show more freedom to describe different observables, would not yield a global description including these new data with an acceptable global $\chi^{2}$. As such our released RnPDFs should be considered as useful and handy tools for observables which are known to be well accounted for by the effects encoded in nPDFs as well as useful exploratory tools for new ones.

\section{ACKNOWLEDGMENTS}

We are grateful to R. Abdul Khalek, N. Armesto, Y. Zhang for very helpful discussions. This project has received funding from the European Union's Horizon 2020 research and innovation program under the Grant Agreement No. 824093 in order to contribute to the EU Virtual Access "NLOAccess" and the French ANR under the Grant No. ANR-20-CE31-0015 ("PrecisOnium"). This work was also partly supported by the French CNRS via the IN2P3 project GLUE@NLO, from the CNRS IEA under the Grant Agreement No. 205210 ("GlueGraph") and "Excitonium", by the Paris-Saclay U. via the P2I Department and by the P2IO Labex via the Gluodynamics project. A. K. is also grateful for the support of Narodowe Centrum Nauki under Grant SONATAbis No. 2019/34/E/ST2/00186.

\section{APPENDIX A: $\chi^{2}$ VALUES FOR THE CONSIDERED DATA}

In this Appendix we provide the values of $\chi^{2}$ for the data sets used in the current analysis which we define as: $\chi^{2}=\Sigma_{i}\left(\frac{T_{i}-D_{i}}{\delta_{i}^{\text {uncort. }}}\right)^{2}$, with $T_{i}$ being the theoretical prediction, $D_{i}$ the central value of the experimental data, and $\delta_{i}^{\text {uncorr. }}$ the uncorrelated experimental uncertainty. The values were already partly available as a supplementary material in Ref. [14], see https://journals.aps.org/prl/supplemental/10 .1103/PhysRevLett.121.052004/appendix.pdf. Specifically, in Table II we present comparison of $\chi^{2}$ values before and after the reweighting for data sets directly used in the reweighting of Ref. [14], and in Table III the values for the selected new data sets that were used in Sec. IV as a crosscheck of the universality of the obtained nPDFs.

\section{APPENDIX B: CROSS CHECKS OF THE REWEIGHTED PDFs}

In this Appendix we provide a selection of additional plots showing the HF RnPDFs that give additional details on the obtained results and can be also compared with the results obtained in Ref. [14]. We show results for the nCTEQ15 and EPPS16 $D, B \rightarrow J / \psi$, and $J / \psi$ RnPDFs. We restrict from showing the results with $\Upsilon(1 S)$ data as (due to the large uncertainties of the data) the impact on the original nPDFs was very limited (see Ref. [14]). In Figs. 17, 18 , and 19, we present gluon NMF obtained from the reweightings with $D, B \rightarrow J / \psi$, and $J / \psi$ meson data correspondingly. Figure (a) always corresponds to the 
TABLE II. Comparison of $\chi^{2}$ values before and after reweighting for the data that were used in the reweighting procedure.

\begin{tabular}{|c|c|c|c|c|c|c|c|}
\hline & & & \multirow[b]{2}{*}{ Original } & \multicolumn{4}{|c|}{ Reweighted } \\
\hline & & & & $D^{0}$ & $J / \psi$ & $B \rightarrow J / \psi$ & $\Upsilon(1 S)$ \\
\hline LHC $J / \psi\left(N_{\text {data }}=71\right)$ & $\begin{array}{c}\text { nCTEQ15 } \\
\text { EPPS16 }\end{array}$ & $\begin{array}{l}\xi=0.5 \\
\xi=1.0 \\
\xi=2.0 \\
\xi=0.5 \\
\xi=1.0 \\
\xi=2.0\end{array}$ & $\begin{array}{r}131 \\
63 \\
90 \\
62 \\
150 \\
220\end{array}$ & $\begin{array}{l}\cdots \\
\ldots \\
\ldots \\
\cdots \\
\cdots \\
\ldots\end{array}$ & $\begin{array}{l}46 \\
53 \\
46 \\
59 \\
59 \\
59\end{array}$ & $\begin{array}{l}\cdots \\
\cdots \\
\cdots \\
\cdots \\
\cdots \\
\cdots\end{array}$ & $\begin{array}{l}\cdots \\
\cdots \\
\cdots \\
\cdots \\
\cdots \\
\cdots\end{array}$ \\
\hline LHC $D^{0}\left(N_{\text {data }}=38\right)$ & $\begin{array}{c}\text { nCTEQ15 } \\
\text { EPPS16 }\end{array}$ & $\begin{array}{l}\xi=0.5 \\
\xi=1.0 \\
\xi=2.0 \\
\xi=0.5 \\
\xi=1.0 \\
\xi=2.0\end{array}$ & $\begin{array}{r}142 \\
39 \\
63 \\
53 \\
140 \\
218\end{array}$ & $\begin{array}{l}56 \\
56 \\
56 \\
37 \\
37 \\
37\end{array}$ & $\begin{array}{l}\ldots \\
\cdots \\
\cdots \\
\cdots \\
\cdots \\
\cdots\end{array}$ & $\begin{array}{l}\ldots \\
\ldots \\
\ldots \\
\ldots \\
\ldots \\
\ldots\end{array}$ & $\begin{array}{l}\ldots \\
\ldots \\
\ldots \\
\ldots \\
\ldots \\
\ldots\end{array}$ \\
\hline $\mathrm{LHC} B \rightarrow J / \psi\left(N_{\text {data }}=37\right)$ & $\begin{array}{c}\text { nCTEQ15 } \\
\text { EPPS16 }\end{array}$ & $\begin{array}{l}\xi=0.5 \\
\xi=1.0 \\
\xi=2.0 \\
\xi=0.5 \\
\xi=1.0 \\
\xi=2.0\end{array}$ & $\begin{array}{r}39 \\
23 \\
15 \\
9 \\
7 \\
8\end{array}$ & $\begin{array}{l}\cdots \\
\ldots \\
\cdots \\
\cdots \\
\cdots \\
\ldots\end{array}$ & $\begin{array}{l}\cdots \\
\cdots \\
\cdots \\
\cdots \\
\cdots \\
\cdots\end{array}$ & $\begin{array}{c}14 \\
11 \\
9 \\
7 \\
7 \\
7\end{array}$ & $\begin{array}{l}\ldots \\
\ldots \\
\ldots \\
\ldots \\
\cdots \\
\ldots\end{array}$ \\
\hline LHC $\Upsilon(1 S)\left(N_{\text {data }}=12\right)$ & $\begin{array}{c}\text { nCTEQ15 } \\
\text { EPPS16 }\end{array}$ & $\begin{array}{l}\xi=0.5 \\
\xi=1.0 \\
\xi=2.0 \\
\xi=0.5 \\
\xi=1.0 \\
\xi=2.0\end{array}$ & $\begin{array}{l}14 \\
11 \\
11 \\
10 \\
10 \\
11\end{array}$ & $\begin{array}{l}\cdots \\
\ldots \\
\ldots \\
\ldots \\
\cdots \\
\ldots\end{array}$ & $\begin{array}{l}\ldots \\
\ldots \\
\ldots \\
\cdots \\
\cdots \\
\ldots\end{array}$ & $\begin{array}{l}\cdots \\
\cdots \\
\cdots \\
\cdots \\
\cdots \\
\ldots\end{array}$ & $\begin{array}{l}13 \\
11 \\
11 \\
10 \\
10 \\
11\end{array}$ \\
\hline
\end{tabular}

reweighting in case of nCTEQ15 nPDFs and Fig. (b) to the reweighting with the EPPS16 nPDFs. The upper rows of Figs. (a) and (b) always show the 68\% CL results (that can be directly compared to the figures presented in Ref. [14]), the lower rows provide the same results but with PDF uncertainties calculated at $90 \%$ CL. In Figs. 20 and 21 we present results for gluon, up quark, and anti-down quark distributions (the other distributions exhibit analogical features). In these plots we displayed distributions obtained from the reweighting with different scale choice $\left\{\mu_{0}, 2 \mu_{0}, 0.5 \mu_{0}\right\}$ and a distribution where scale uncertainties were combined. For better visibility all PDFs were scaled by the central value of the combined distribution. As expected the main impact is on the gluon distribution, the

TABLE III. Comparison of $\chi^{2}$ values for a selection of the heavy flavor data that were not included in the reweighting procedure.

\begin{tabular}{|c|c|c|c|c|c|c|c|}
\hline & & & \multirow[b]{2}{*}{ Original } & \multicolumn{4}{|c|}{ Reweighted } \\
\hline & & & & $\overline{D^{0}}$ & $J / \psi$ & $B \rightarrow J / \psi$ & $\Upsilon(1 S)$ \\
\hline \multirow{6}{*}{$\begin{array}{l}\text { PHENIX } J / \psi\left(N_{\mathrm{data}}=30\right)[128] \\
\text { Fig. } 5 \text { (forward only) and Fig. } 7\end{array}$} & nCTEQ15 & $\xi=0.5$ & 94.2 & $\ldots$ & 34.5 & $\ldots$ & $\ldots$ \\
\hline & & $\xi=1.0$ & 104.6 & $\ldots$ & 56.0 & $\ldots$ & $\ldots$ \\
\hline & & $\xi=2.0$ & 158.0 & $\ldots$ & 81.7 & $\cdots$ & $\ldots$ \\
\hline & EPPS16 & $\xi=0.5$ & 115.8 & $\ldots$ & 49.2 & $\ldots$ & $\ldots$ \\
\hline & & $\xi=1.0$ & 69.9 & $\ldots$ & 53.5 & $\ldots$ & $\ldots$ \\
\hline & & $\xi=2.0$ & 133.5 & $\ldots$ & 90.8 & $\ldots$ & $\ldots$ \\
\hline \multirow{6}{*}{$\begin{array}{l}\text { LHCb } B\left(N_{\text {data }}=10\right)[135] \\
\text { Fig. } 13 \text { and Fig. } 14\end{array}$} & nCTEQ15 & $\xi=0.5$ & 10.1 & 9.1 & $\ldots$ & $\ldots$ & $\ldots$ \\
\hline & & $\xi=1.0$ & 12.1 & 6.4 & $\ldots$ & $\ldots$ & $\ldots$ \\
\hline & & $\xi=2.0$ & 14.4 & 6.8 & $\ldots$ & $\ldots$ & $\ldots$ \\
\hline & EPPS16 & $\xi=0.5$ & 6.8 & 9.6 & $\ldots$ & $\ldots$ & $\ldots$ \\
\hline & & $\xi=1.0$ & 5.9 & 6.6 & $\ldots$ & $\ldots$ & $\ldots$ \\
\hline & & $\xi=2.0$ & 7.1 & 7.1 & $\ldots$ & $\ldots$ & $\ldots$ \\
\hline
\end{tabular}



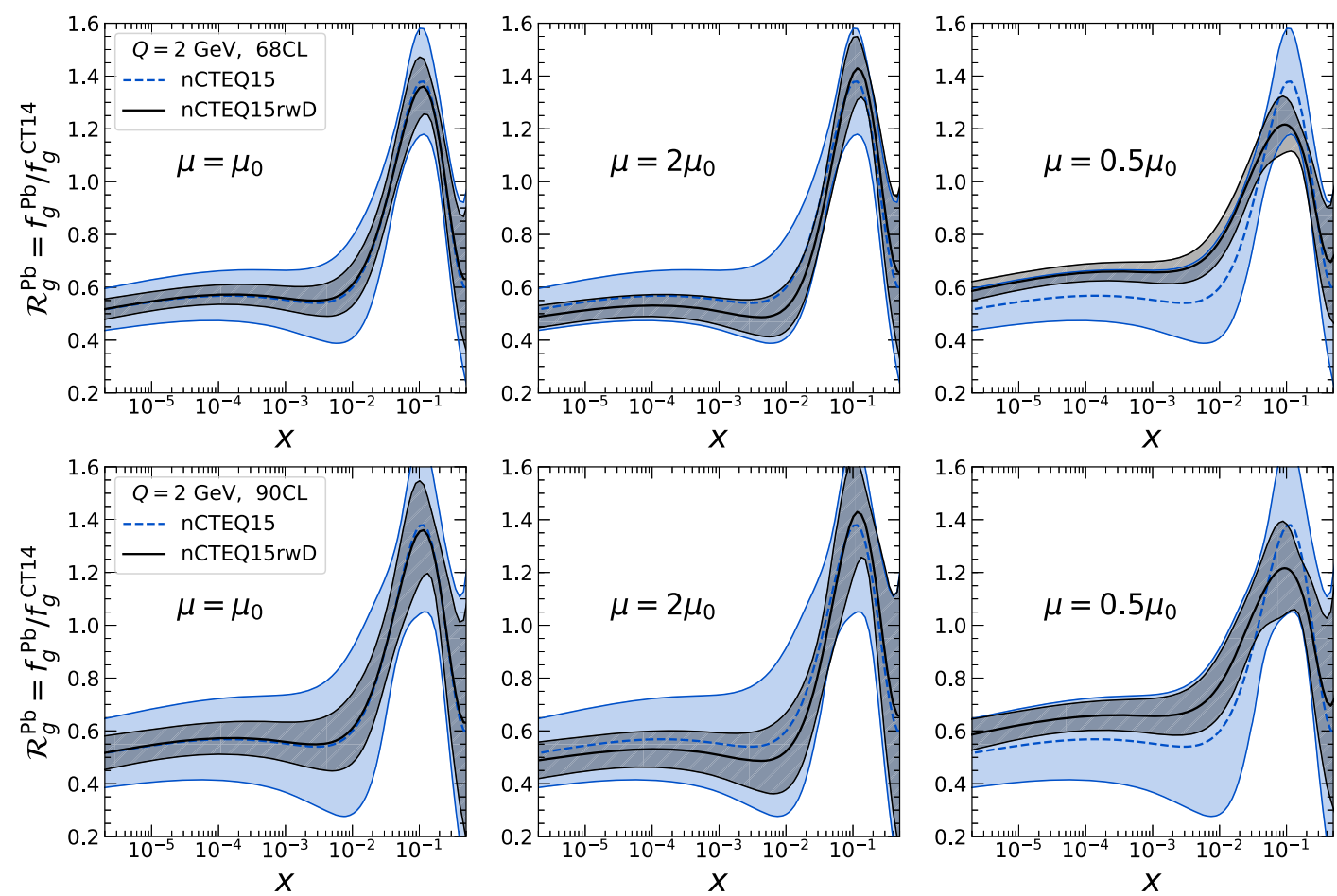

(a)
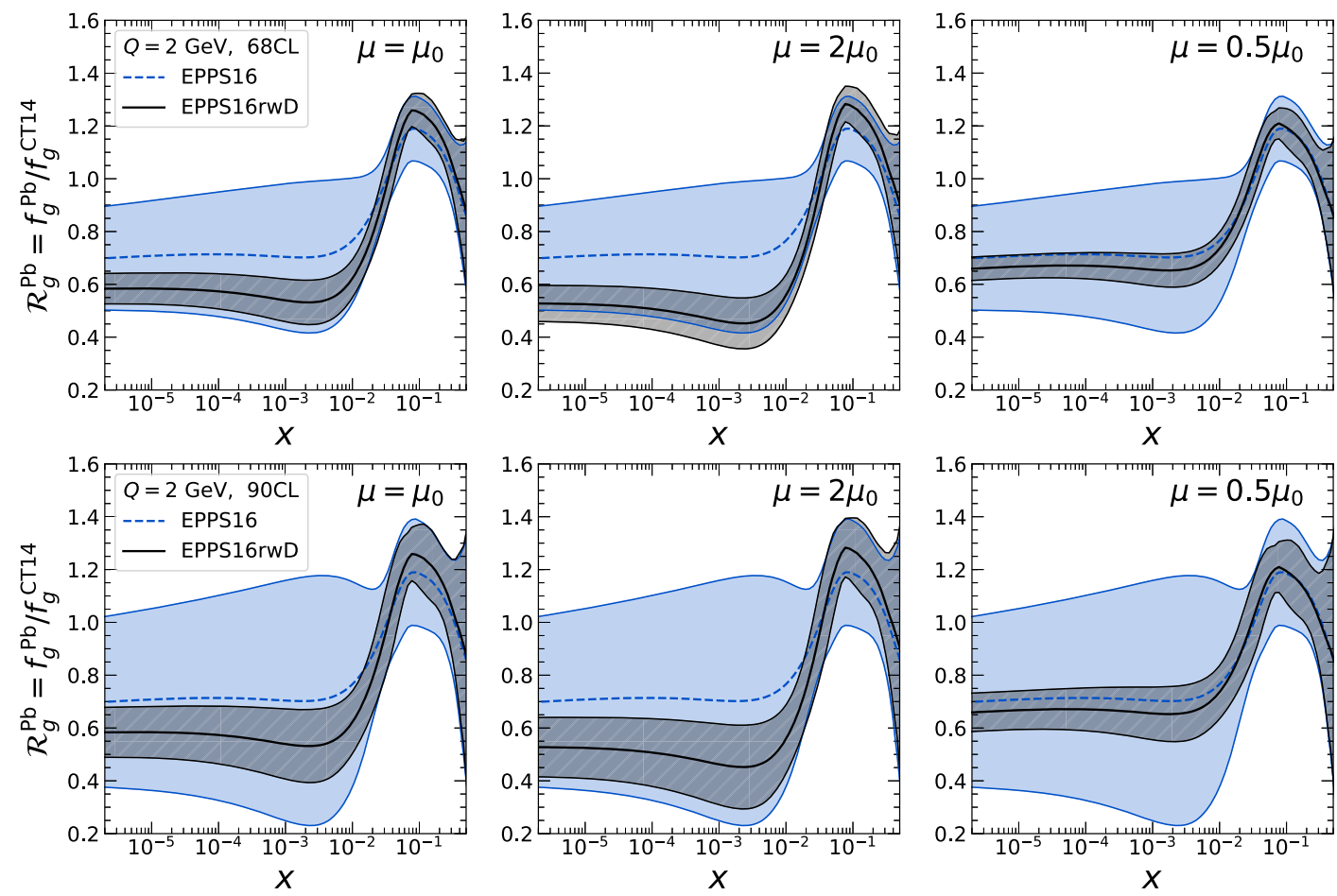

(b)

FIG. 17. Gluon distribution resulting from reweighting of (a) nCTEQ15 and (b) EPPS16 nPDFs with $D$-meson data. The upper rows show errors at $68 \%$ CL for comparison with Fig. 1(f) in the original reweighting paper [14]. The lower rows show the same distributions with errors at $90 \%$ CL. 

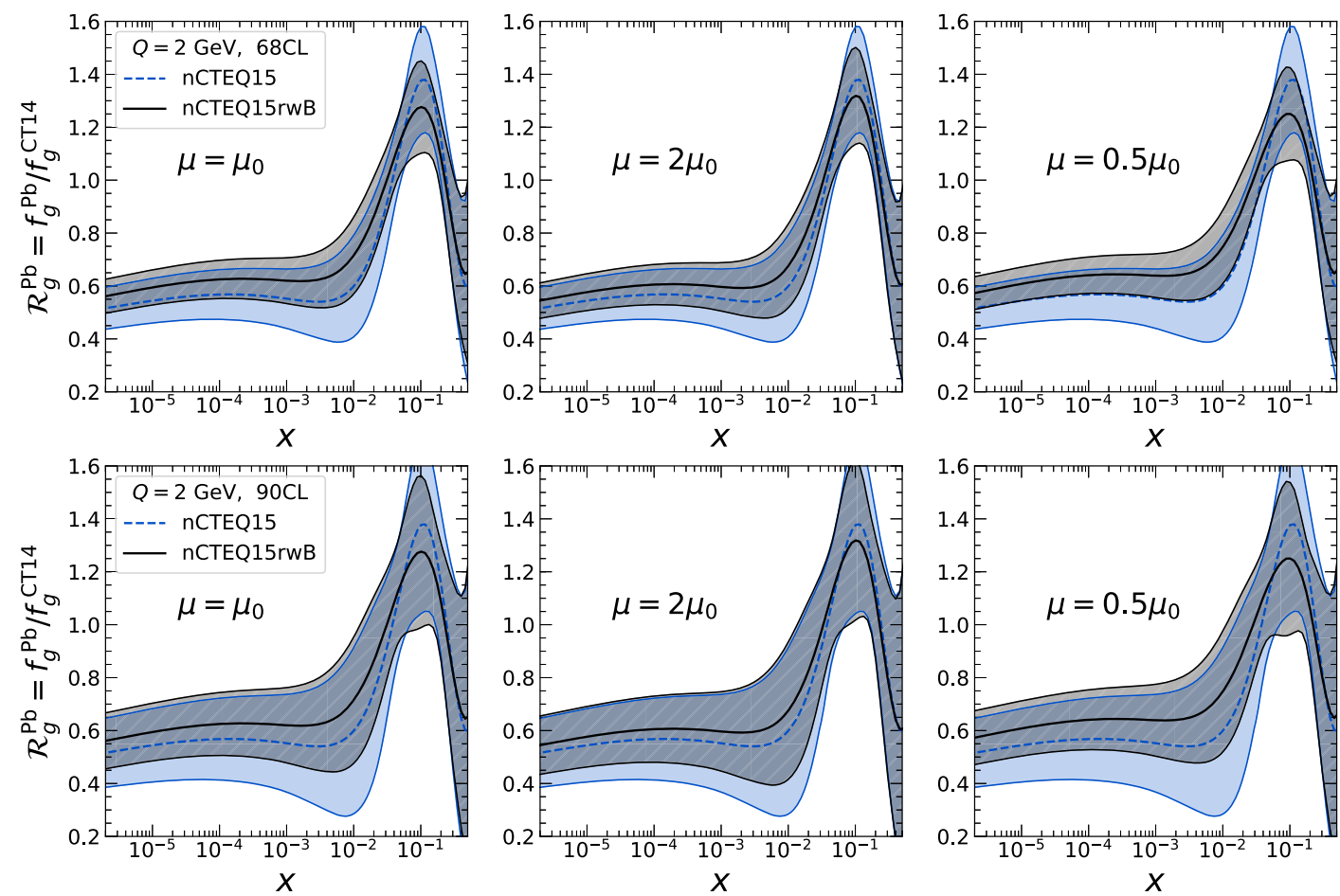

(a)
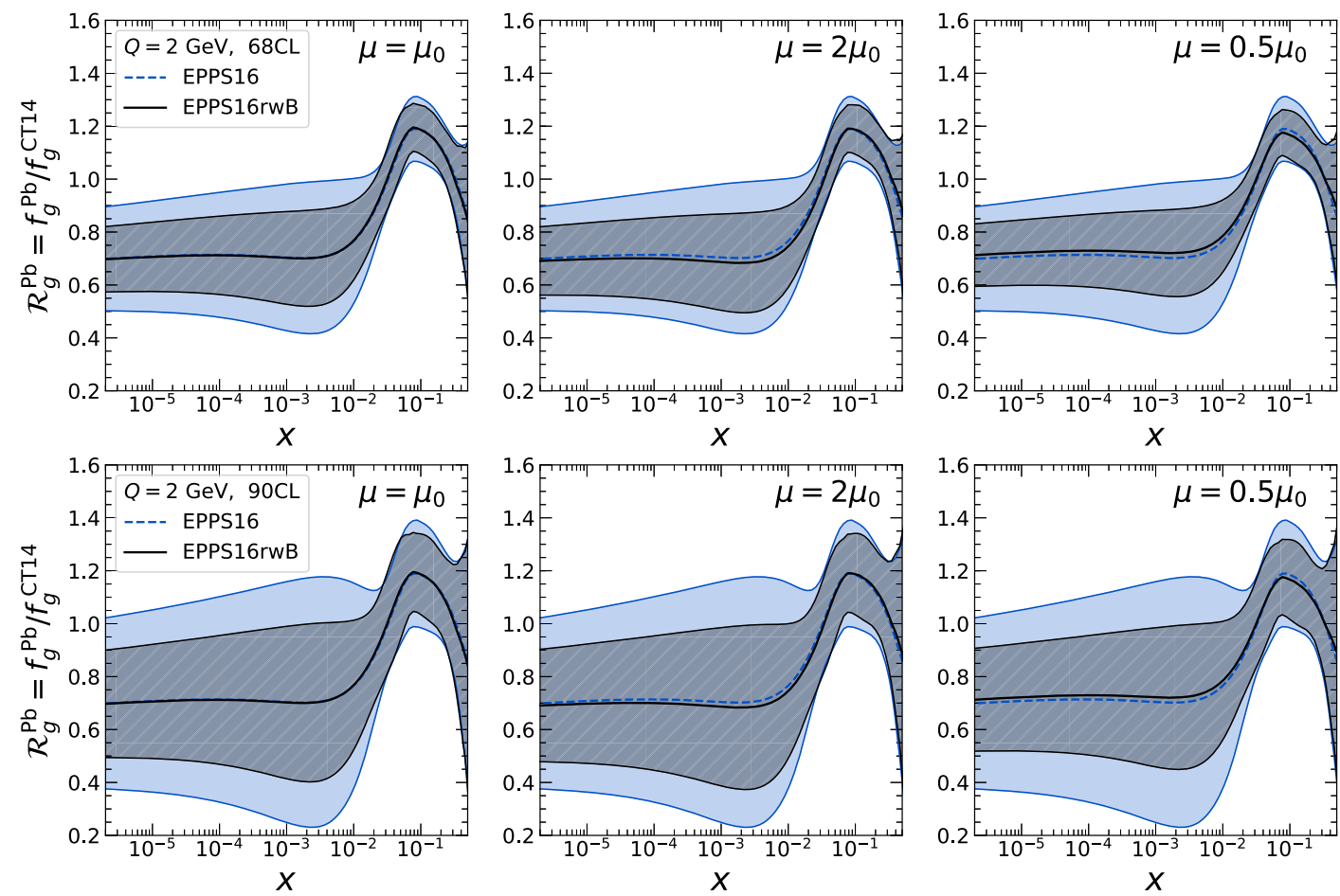

(b)

FIG. 18. Gluon distribution resulting from reweighting of (a) nCTEQ15 and (b) EPPS16 nPDFs with $B \rightarrow J / \psi$ data. The upper rows show errors at $68 \% \mathrm{CL}$ for comparison with Fig. 1(f) in the original reweighting paper [14]. The lower rows show the same distributions with errors at $90 \%$ CL. 

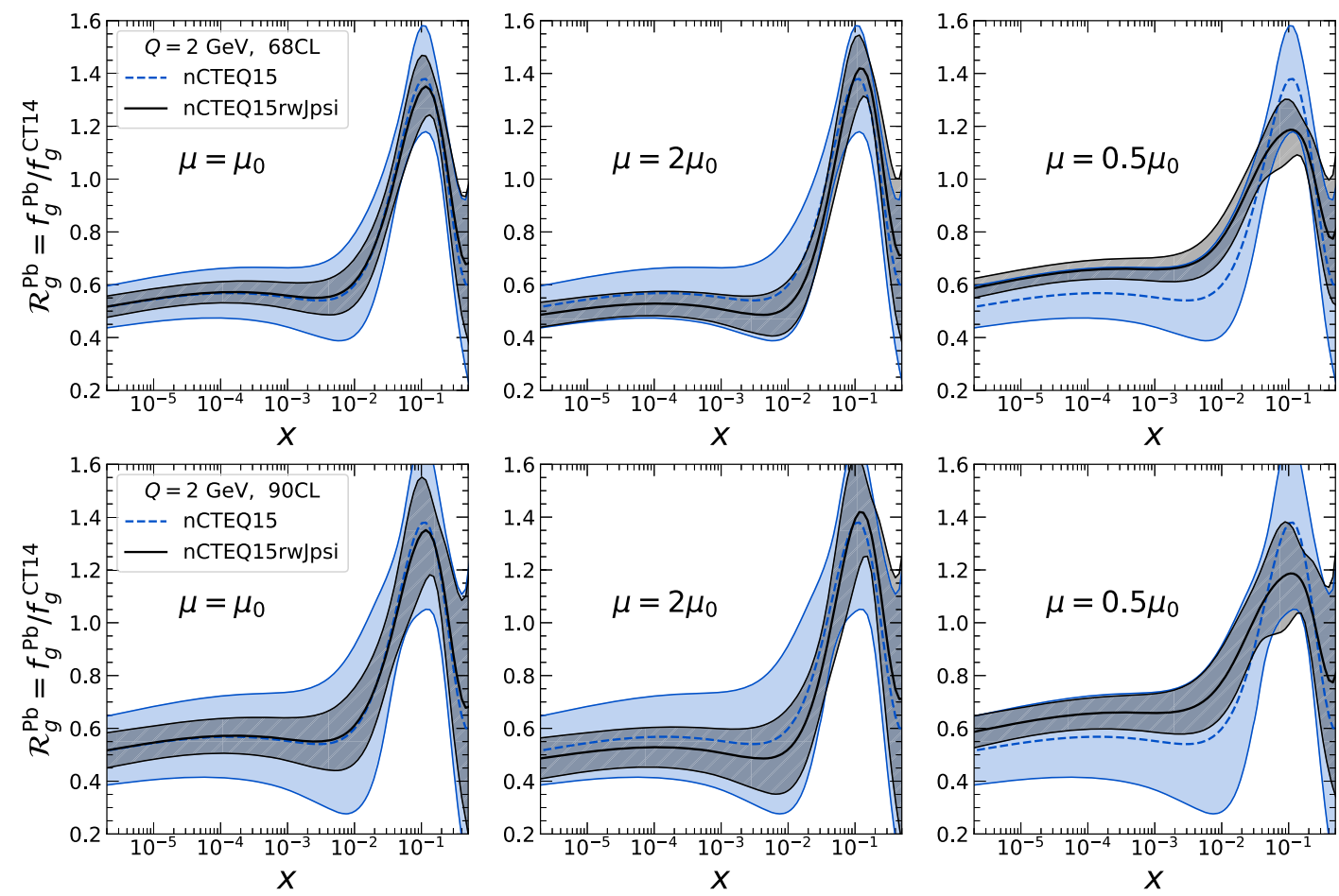

(a)
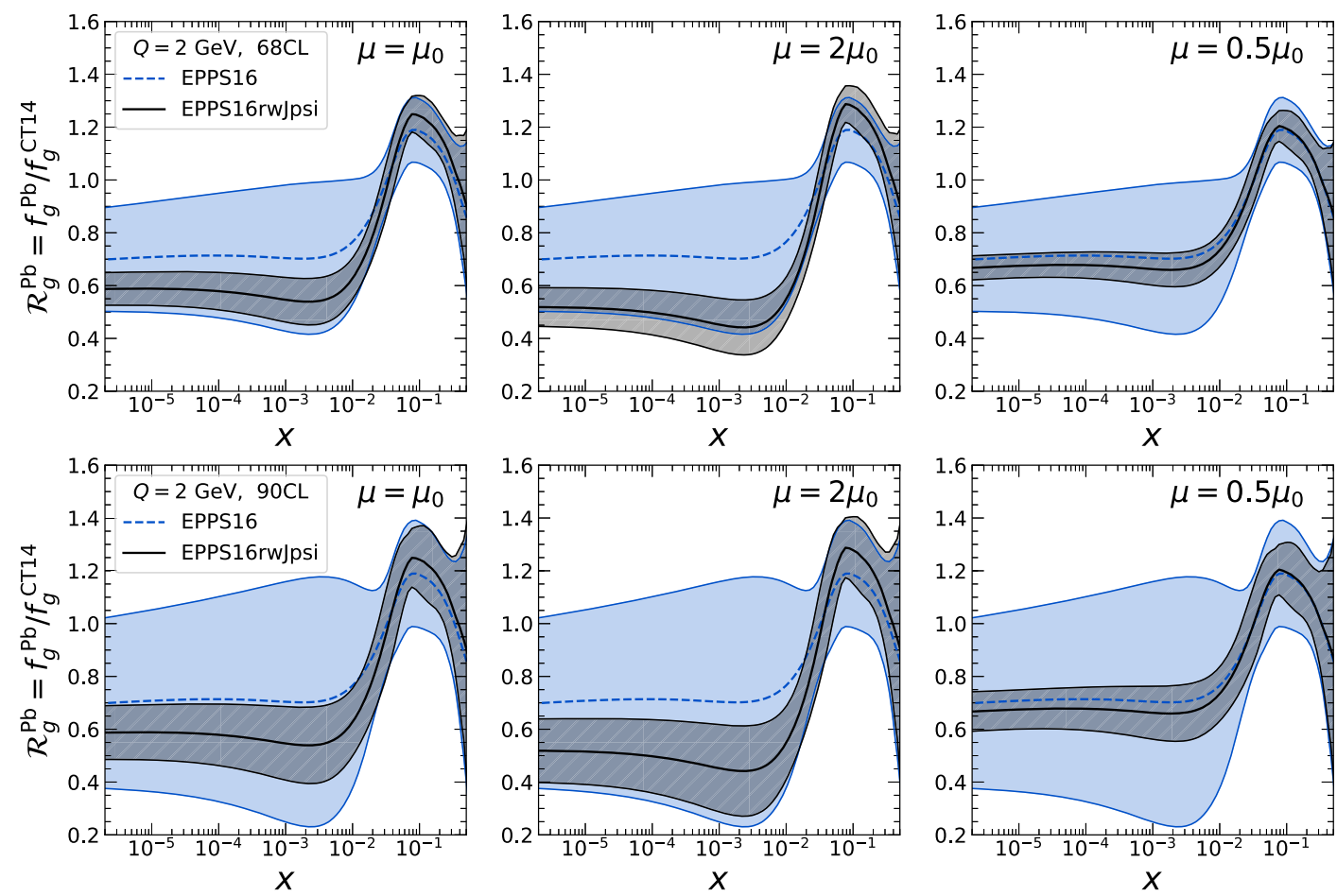

(b)

FIG. 19. Gluon distribution resulting from reweighting of (a) nCTEQ15 and (b) EPPS16 nPDFs with $J / \psi$ data. The upper rows show errors at $68 \%$ CL for comparison with Fig. 1(f) in the original reweighting paper [14]. The lower rows show the same distributions with errors at $90 \% \mathrm{CL}$. 

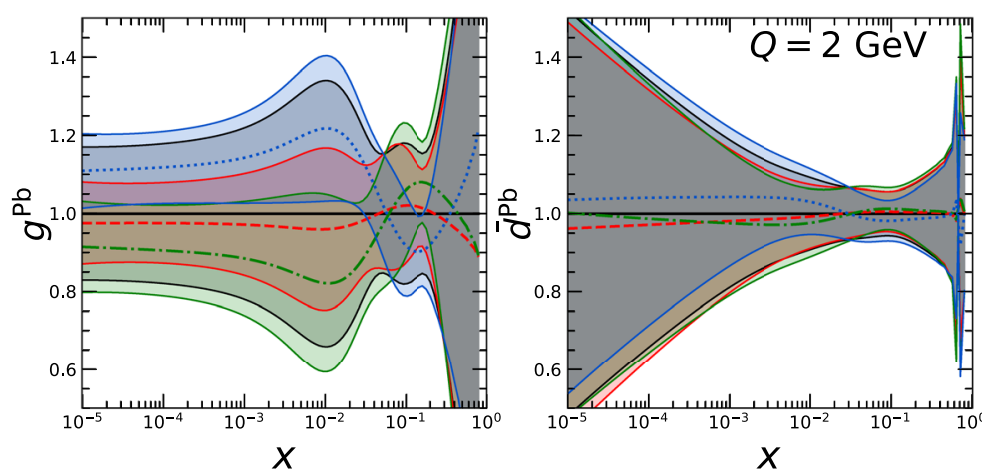

(a)
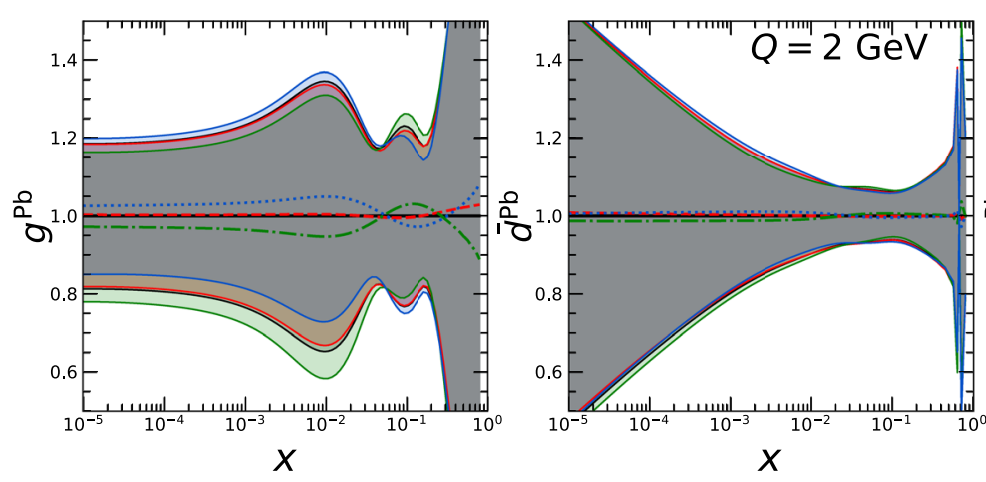

(b)
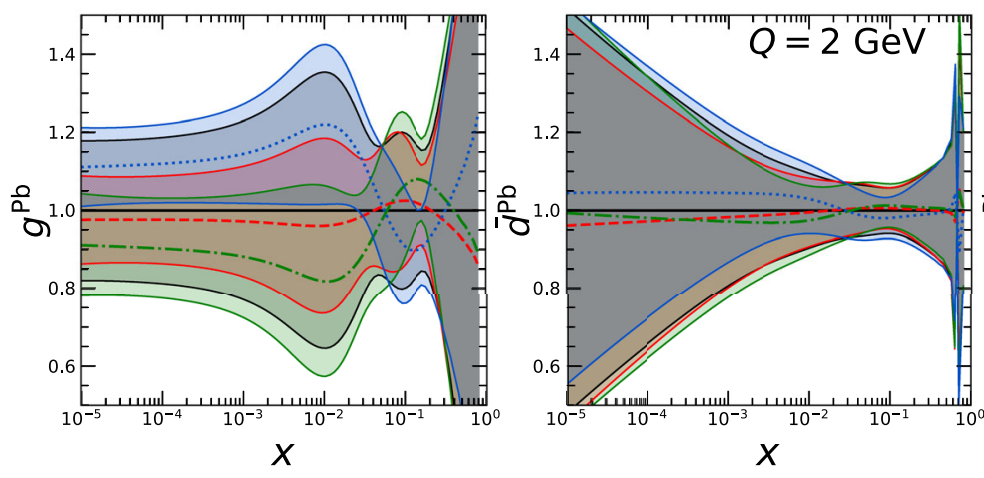

(c)
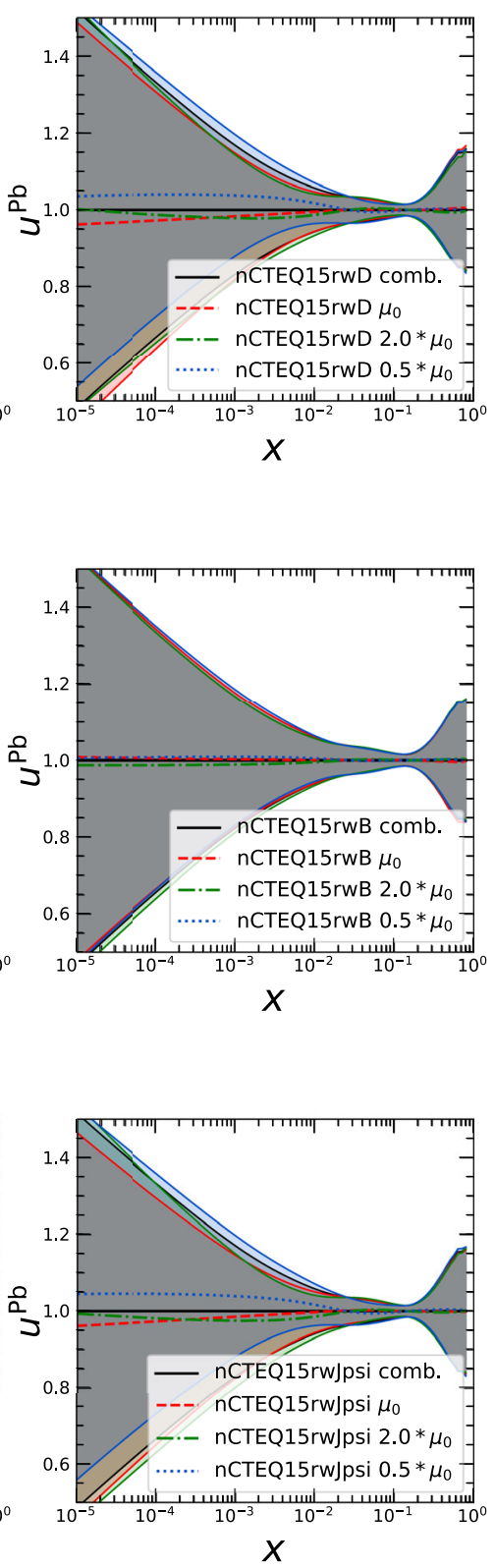

FIG. 20. Comparison of the reweighting results for nCTEQ15 nPDFs with (a) $D$-meson, (b) $B \rightarrow J / \psi$, and (c) $J / \psi$ data using different choices of factorization scales. Additionally we show a combined set of PDFs for uncertainties from different scales choices were combined (solid black line).

quark PDFs are mostly unchanged after including the HF data. We can also see that the uncertainty of the set with the combined scale uncertainty is smaller than the envelope of the error bands provided by the results for individual scale choices but larger than the uncertainty of the central scale choice. Generally whenever possible we recommend to use
PDF sets obtained with specific scale choice (such that it is correlated with the scale used in the theoretical calculation). For observables where there is no reason to correlate the scales one can either take the envelope of the three PDF sets or use the combined PDF set to restrict the number of necessary evaluations. 

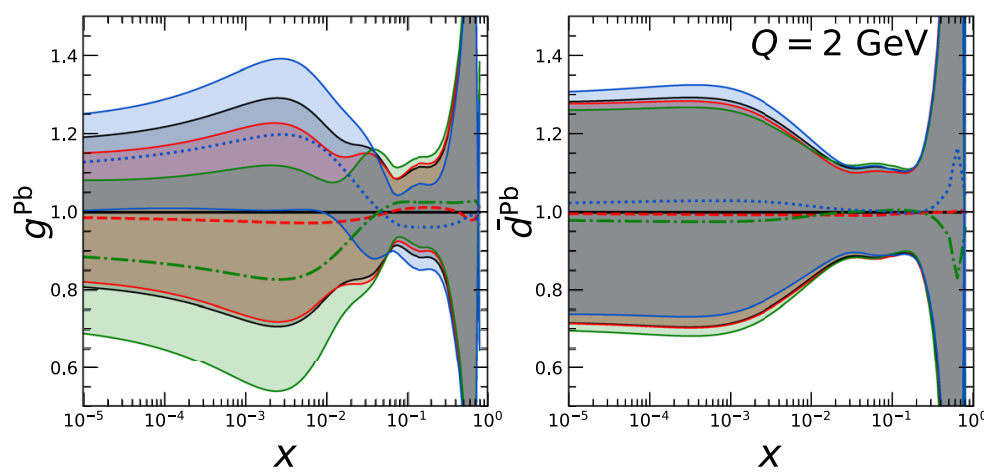

(a)
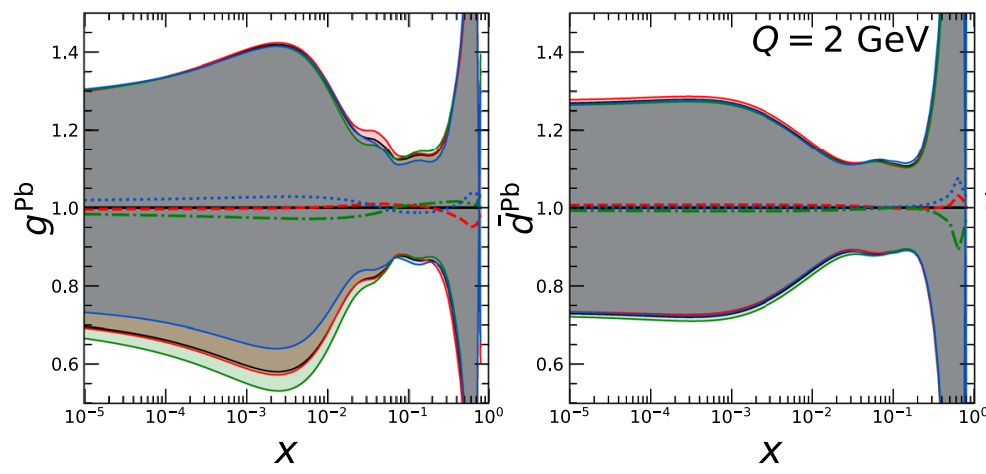

(b)
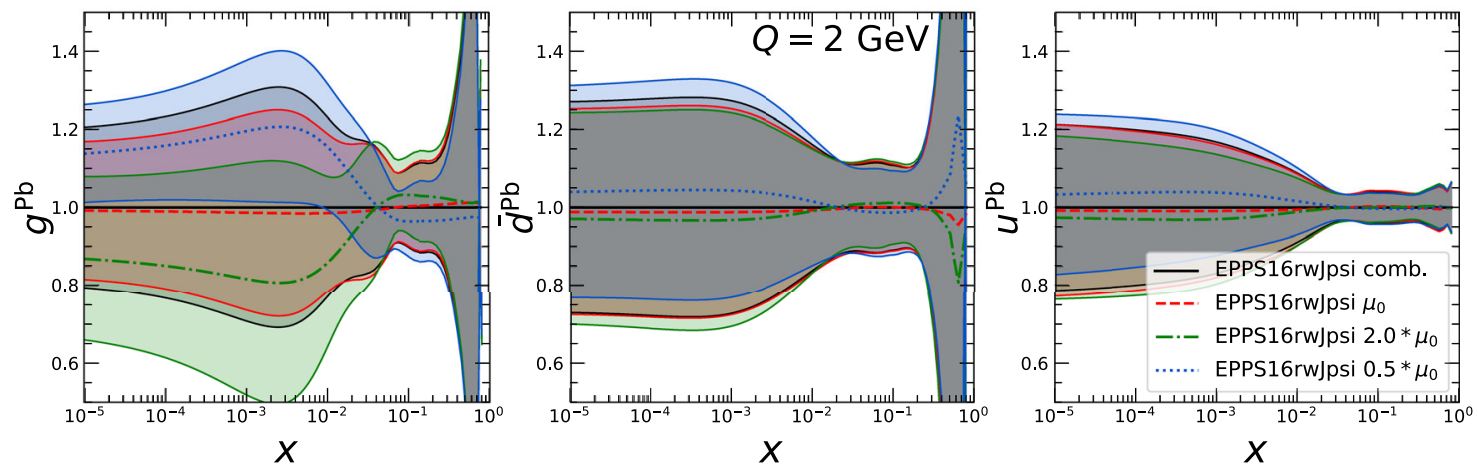

(c)
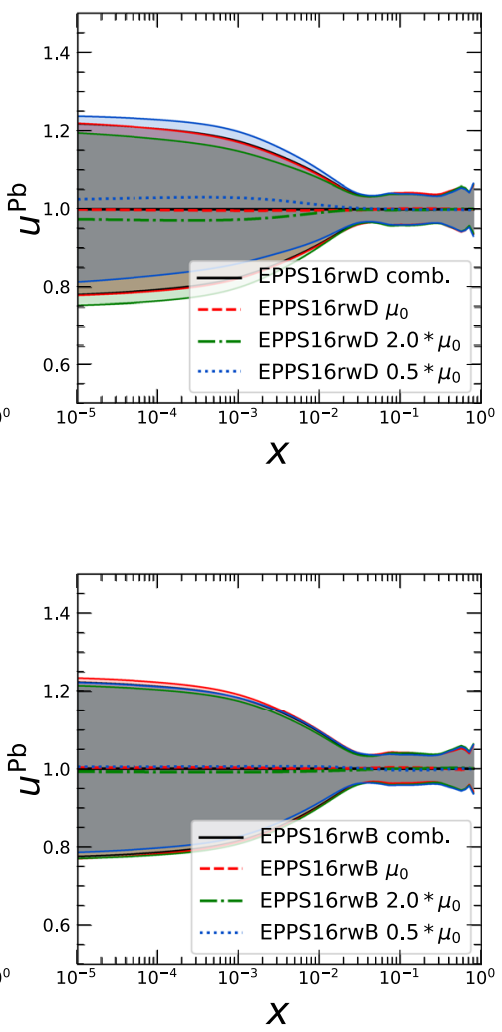

$X$

FIG. 21. Comparison of the reweighting results for EPPS16 nPDFs with (a) $D$-meson, (b) $B \rightarrow J / \psi$, and (c) $J / \psi$ data using different choices of factorization scales. Additionally we show a combined set of PDFs for uncertainties from different scales choices were combined (solid black line).

[1] M. Arneodo et al. (New Muon Collaboration), The Q**2 dependence of the structure function ratio $\mathrm{F} 2 \mathrm{Sn} / \mathrm{F} 2 \mathrm{C}$ and the difference $\mathrm{R} \mathrm{Sn}-\mathrm{R} \mathrm{C}$ in deep inelastic muon scattering, Nucl. Phys. B481, 23 (1996).

[2] A. M. Sirunyan et al. (CMS Collaboration), Observation of nuclear modifications in $W^{ \pm}$boson production in $\mathrm{pPb}$ collisions at $\sqrt{s_{\mathrm{NN}}}=8.16 \mathrm{TeV}$, Phys. Lett. B 800, 135048 (2020).
[3] G. Aad et al. (ATLAS Collaboration), $Z$ boson production in $p+\mathrm{Pb}$ collisions at $\sqrt{s_{\mathrm{NN}}}=5.02 \mathrm{TeV}$ measured with the ATLAS detector, Phys. Rev. C 92, 044915 (2015).

[4] V. Khachatryan et al. (CMS Collaboration), Study of W boson production in $\mathrm{pPb}$ collisions at $\sqrt{s_{N N}}=5.02 \mathrm{TeV}$, Phys. Lett. B 750, 565 (2015). 
[5] V. Khachatryan et al. (CMS Collaboration), Study of Z boson production in $\mathrm{pPb}$ collisions at $\sqrt{s_{N N}}=5.02 \mathrm{TeV}$, Phys. Lett. B 759, 36 (2016).

[6] J. Adam et al. (ALICE Collaboration), W and $\mathrm{Z}$ boson production in $\mathrm{p}-\mathrm{Pb}$ collisions at $\sqrt{s_{N N}}=5.02 \mathrm{TeV}$, J. High Energy Phys. 02 (2017) 077.

[7] R. Aaij et al. (LHCb Collaboration), Observation of $Z$ production in proton-lead collisions at LHCb, J. High Energy Phys. 09 (2014) 030.

[8] S. Chatrchyan et al. (CMS Collaboration), Studies of dijet transverse momentum balance and pseudorapidity distributions in $\mathrm{pPb}$ collisions at $\sqrt{s_{\mathrm{NN}}}=5.02 \mathrm{TeV}$, Eur. Phys. J. C 74, 2951 (2014).

[9] K. J. Eskola, P. Paakkinen, H. Paukkunen, and C. A. Salgado, EPPS16: Nuclear parton distributions with LHC data, Eur. Phys. J. C 77, 163 (2017).

[10] M. Aaboud et al. (ATLAS Collaboration), Measurement of prompt photon production in $\sqrt{s_{\mathrm{NN}}}=8.16 \mathrm{TeV} p+\mathrm{Pb}$ collisions with ATLAS, Phys. Lett. B 796, 230 (2019).

[11] R. A. Khalek, J. J. Ethier, J. Rojo, and G. van Weelden, nNNPDF2.0: Quark flavor separation in nuclei from LHC data, J. High Energy Phys. 09 (2020) 183.

[12] S. Acharya et al. (ALICE Collaboration), Neutral pion and $\eta$ meson production in $\mathrm{p}-\mathrm{Pb}$ collisions at $\sqrt{s_{\mathrm{NN}}}=$ 5.02 TeV, Eur. Phys. J. C 78, 624 (2018).

[13] J. Adam et al. (ALICE Collaboration), Multiplicity dependence of charged pion, kaon, and (anti)proton production at large transverse momentum in $\mathrm{p}-\mathrm{Pb}$ collisions at $\sqrt{s_{\mathrm{NN}}}=5.02 \mathrm{TeV}$, Phys. Lett. B 760, 720 (2016).

[14] A. Kusina, J.-P. Lansberg, I. Schienbein, and H.-S. Shao, Gluon Shadowing in Heavy-Flavor Production at the LHC, Phys. Rev. Lett. 121, 052004 (2018).

[15] J.-P. Lansberg, New observables in inclusive production of quarkonia, Phys. Rep. 889, 1 (2020).

[16] A. Andronic et al., Heavy-flavor and quarkonium production in the LHC era: From proton-proton to heavy-ion collisions, Eur. Phys. J. C 76, 107 (2016).

[17] N. Brambilla et al., Heavy quarkonium: Progress, puzzles, and opportunities, Eur. Phys. J. C 71, 1534 (2011).

[18] R. A. Khalek et al., Science requirements and detector concepts for the electron-ion collider: EIC yellow report, arXiv:2103.05419.

[19] R. A. Khalek, J. J. Ethier, E. R. Nocera, and J. Rojo, Selfconsistent determination of proton and nuclear PDFs at the Electron Ion Collider, Phys. Rev. D 103, 096005 (2021).

[20] H.-S. Shao, Probing impact-parameter dependent nuclear parton densities from double parton scatterings in heavyion collisions, Phys. Rev. D 101, 054036 (2020).

[21] H.-S. Shao, J/ $\psi$ meson production in association with an open charm hadron at the LHC: A reappraisal, Phys. Rev. D 102, 034023 (2020).

[22] K. Kovarik et al., nCTEQ15-Global analysis of nuclear parton distributions with uncertainties in the CTEQ framework, Phys. Rev. D 93, 085037 (2016).

[23] V. N. Gribov and L.N. Lipatov, Deep inelastic e p scattering in perturbation theory, Yad. Fiz. 15, 781 (1972) [Sov. J. Nucl. Phys. 15, 438 (1972)].

[24] G. Altarelli and G. Parisi, Asymptotic freedom in parton language, Nucl. Phys. B126, 298 (1977).
[25] Y. L. Dokshitzer, Calculation of the Structure Functions for Deep Inelastic Scattering and e+ e- Annihilation by Perturbation Theory in Quantum Chromodynamics, Zh. Eksp. Teor. Fiz. 73, 1216 (1977) [Sov. Phys. JETP 46, 641 (1977)].

[26] B. Schmookler et al. (CLAS Collaboration), Modified structure of protons and neutrons in correlated pairs, Nature (London) 566, 354 (2019).

[27] M. Walt, I. Helenius, and W. Vogelsang, Open-source QCD analysis of nuclear parton distribution functions at NLO and NNLO, Phys. Rev. D 100, 096015 (2019).

[28] A. Kusina et al., Impact of LHC vector boson production in heavy ion collisions on strange PDFs, Eur. Phys. J. C 80, 968 (2020).

[29] H. Khanpour, M. Soleymaninia, S. A. Tehrani, H. Spiesberger, and V. Guzey, Nuclear parton distribution functions with uncertainties in the general mass variable flavor number scheme, arXiv:2010.00555.

[30] E. P. Segarra et al., nCTEQ15HIX-Extending nPDF analyses into the high- $x$, Low $Q^{2}$ region, Phys. Rev. D 103, 114015 (2021).

[31] T.-J. Hou et al., New CTEQ global analysis of quantum chromodynamics with high-precision data from the LHC, Phys. Rev. D 103, 014013 (2021).

[32] L. A. Harland-Lang, A. D. Martin, P. Motylinski, and R. S. Thorne, Parton distributions in the LHC era: MMHT 2014 PDFs, Eur. Phys. J. C 75, 204 (2015).

[33] R. D. Ball et al. (NNPDF Collaboration), Parton distributions from high-precision collider data, Eur. Phys. J. C 77, 663 (2017).

[34] A. Accardi, L. T. Brady, W. Melnitchouk, J. F. Owens, and N. Sato, Constraints on large- $x$ parton distributions from new weak boson production and deep-inelastic scattering data, Phys. Rev. D 93, 114017 (2016).

[35] S. Alekhin, J. Blümlein, S. Moch, and R. Placakyte, Parton distribution functions, $\alpha_{s}$, and heavy-quark masses for LHC Run II, Phys. Rev. D 96, 014011 (2017).

[36] S. Alekhin et al., HERAFitter, Eur. Phys. J. C 75, 304 (2015).

[37] J. J. Aubert et al. (European Muon Collaboration), The ratio of the nucleon structure functions $F 2_{n}$ for iron and deuterium, Phys. Lett. 123B, 275 (1983).

[38] M. S. Goodman et al., Observation of Shadowing in the Virtual Photon Total Hadronic Cross-Section on Nuclei, Phys. Rev. Lett. 47, 293 (1981).

[39] A. Bodek et al., A Comparison of the Deep Inelastic Structure Functions of Deuterium and Aluminum Nuclei, Phys. Rev. Lett. 51, 534 (1983).

[40] G. Bari et al. (BCDMS Collaboration), A measurement of nuclear effects in deep inelastic muon scattering on deuterium, nitrogen and iron targets, Phys. Lett. 163B, 282 (1985).

[41] A. C. Benvenuti et al. (BCDMS Collaboration), Nuclear effects in deep inelastic muon scattering on deuterium and iron targets, Phys. Lett. B 189, 483 (1987).

[42] J. Ashman et al. (European Muon Collaboration), Measurement of the ratios of deep inelastic muon-nucleus cross-sections on various nuclei compared to deuterium, Phys. Lett. B 202, 603 (1988). 
[43] M. Arneodo et al. (European Muon Collaboration), Shadowing in deep inelastic muon scattering from nuclear targets, Phys. Lett. B 211, 493 (1988).

[44] D. Higinbotham, G. A. Miller, O. Hen, and K. Rith, The EMC effect still puzzles after 30 years, CERN Courier 53N4, 24 (2013).

[45] D. F. Geesaman, K. Saito, and A. W. Thomas, The nuclear EMC effect, Annu. Rev. Nucl. Part. Sci. 45, 337 (1995).

[46] H. Khanpour and S. A. Tehrani, Global analysis of nuclear parton distribution functions and their uncertainties at nextto-next-to-leading order, Phys. Rev. D 93, 014026 (2016).

[47] D. de Florian, R. Sassot, P. Zurita, and M. Stratmann, Global analysis of nuclear parton distributions, Phys. Rev. D 85, 074028 (2012).

[48] M. Hirai, S. Kumano, and T. H. Nagai, Determination of nuclear parton distribution functions and their uncertainties in next-to-leading order, Phys. Rev. C 76, 065207 (2007).

[49] T. Stavreva, I. Schienbein, F. Arleo, K. Kovarik, F. Olness, J. Y. Yu, and J. F. Owens, Probing gluon and heavy-quark nuclear PDFs with gamma $+Q$ production in $\mathrm{pA}$ collisions, J. High Energy Phys. 01 (2011) 152.

[50] I. Helenius, H. Paukkunen, and N. Armesto, nPDF constraints from the large hadron electron collider, Proc. Sci., DIS2016 (2016) 276 [arXiv:1606.09003].

[51] K. J. Eskola, H. Paukkunen, and C. A. Salgado, EPS09: A new generation of NLO and LO nuclear parton distribution functions, J. High Energy Phys. 04 (2009) 065.

[52] O. Zenaiev et al. (PROSA Collaboration), Impact of heavy-flavor production cross sections measured by the $\mathrm{LHCb}$ experiment on parton distribution functions at low x, Eur. Phys. J. C 75, 396 (2015).

[53] R. Gauld, J. Rojo, L. Rottoli, and J. Talbert, Charm production in the forward region: constraints on the small-x gluon and backgrounds for neutrino astronomy, J. High Energy Phys. 11 (2015) 009.

[54] M. Cacciari, M. L. Mangano, and P. Nason, Gluon PDF constraints from the ratio of forward heavy-quark production at the LHC at $\sqrt{S}=7$ and $13 \mathrm{TeV}$, Eur. Phys. J. C 75, 610 (2015).

[55] R. Gauld and J. Rojo, Precision Determination of the Small- $x$ Gluon from Charm Production at LHCb, Phys. Rev. Lett. 118, 072001 (2017).

[56] E. G. de Oliveira, A. D. Martin, and M. G. Ryskin, Low x gluons determined by open charm production, arXiv: 1705.08845 .

[57] C. Gerschel and J. Hufner, A contribution to the suppression of the $\mathrm{J} / \mathrm{psi}$ meson produced in high-energy nucleus nucleus collisions, Phys. Lett. B 207, 253 (1988).

[58] R. Vogt, $J / \psi$ production and suppression, Phys. Rep. 310, 197 (1999).

[59] E. G. Ferreiro, Excited charmonium suppression in proton-nucleus collisions as a consequence of comovers, Phys. Lett. B 749, 98 (2015).

[60] E. G. Ferreiro and J.-P. Lansberg, Is bottomonium suppression in proton-nucleus and nucleus-nucleus collisions at LHC energies due to the same effects?, J. High Energy Phys. 10 (2018) 094; 03 (2019) 063(E).

[61] A. Capella and E. G. Ferreiro, J/ psi suppression at $s * *(1 / 2)=200-\mathrm{GeV}$ in the comovers interaction model, Eur. Phys. J. C 42, 419 (2005).
[62] A. Capella, E. G. Ferreiro, and A. B. Kaidalov, Nonsaturation of the J/psi Suppression at Large Transverse Energy in the Comovers Approach, Phys. Rev. Lett. 85, 2080 (2000).

[63] S. Gavin and R. Vogt, $J / \psi$ suppression from hadronnucleus to nucleus-nucleus collisions, Nucl. Phys. B345, 104 (1990).

[64] S. J. Brodsky and P. Hoyer, The Nucleus as a Color Filter in QCD Decays: Hadroproduction in Nuclei, Phys. Rev. Lett. 63, 1566 (1989).

[65] B. Ducloué, T. Lappi, and H. Mäntysaari, Forward $J / \psi$ production in proton-nucleus collisions at high energy, Phys. Rev. D 91, 114005 (2015).

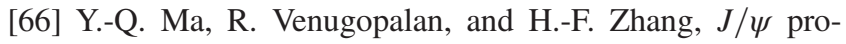
duction and suppression in high energy proton-nucleus collisions, Phys. Rev. D 92, 071901 (2015).

[67] H. Fujii and K. Watanabe, Heavy quark pair production in high energy pA collisions: Quarkonium, Nucl. Phys. A915, 1 (2013).

[68] J.-W. Qiu, P. Sun, B.-W. Xiao, and F. Yuan, Universal suppression of heavy quarkonium production in $\mathrm{pA}$ collisions at low transverse momentum, Phys. Rev. D 89, 034007 (2014).

[69] B. Kopeliovich, A. Tarasov, and J. Hufner, Coherence phenomena in charmonium production off nuclei at the energies of RHIC and LHC, Nucl. Phys. A696, 669 (2001).

[70] E. G. Ferreiro, F. Fleuret, J. P. Lansberg, and A. Rakotozafindrabe, Cold nuclear matter effects on $\mathrm{J} / \mathrm{psi}$ production: Intrinsic and extrinsic transverse momentum effects, Phys. Lett. B 680, 50 (2009).

[71] E. G. Ferreiro, F. Fleuret, J. P. Lansberg, N. Matagne, and A. Rakotozafindrabe, Upsilon production in $\mathrm{p}(\mathrm{d}) \mathrm{A}$ collisions at RHIC and the LHC, Eur. Phys. J. C 73, 2427 (2013).

[72] E. G. Ferreiro, F. Fleuret, J. P. Lansberg, and A. Rakotozafindrabe, Impact of the nuclear modification of the gluon densities on $J / \psi$ production in $p \mathrm{~Pb}$ collisions at $\sqrt{s_{\mathrm{NN}}}=5 \mathrm{TeV}$, Phys. Rev. C 88, 047901 (2013).

[73] R. Vogt, Cold nuclear matter effects on $J / \psi$ and $\mathrm{r}$ production at the LHC, Phys. Rev. C 81, 044903 (2010).

[74] J. L. Albacete et al., Predictions for $p+\mathrm{Pb}$ collisions at $\sqrt{s_{\mathrm{NN}}}=5 \mathrm{TeV}$ : Comparison with data, Int. J. Mod. Phys. E 25, 1630005 (2016).

[75] J. L. Albacete et al., Predictions for cold nuclear matter effects in $p+\mathrm{Pb}$ collisions at $\sqrt{s_{\mathrm{NN}}}=8.16 \mathrm{TeV}$, Nucl. Phys. A972, 18 (2018).

[76] J.-P. Lansberg and H.-S. Shao, Towards an automated tool to evaluate the impact of the nuclear modification of the gluon density on quarkonium, D and B meson production in proton-nucleus collisions, Eur. Phys. J. C 77, 1 (2017).

[77] H.-S. Shao, HELAC-Onia: An automatic matrix element generator for heavy quarkonium physics, Comput. Phys. Commun. 184, 2562 (2013).

[78] H.-S. Shao, HELAC-Onia 2.0: An upgraded matrixelement and event generator for heavy quarkonium physics, Comput. Phys. Commun. 198, 238 (2016).

[79] B. A. Kniehl, G. Kramer, I. Schienbein, and H. Spiesberger, Inclusive $D^{* \pm}$ production in $\mathrm{p}$ anti-p collisions with massive charm quarks, Phys. Rev. D 71, 014018 (2005). 
[80] B. A. Kniehl, G. Kramer, I. Schienbein, and H. Spiesberger, Collinear subtractions in hadroproduction of heavy quarks, Eur. Phys. J. C 41, 199 (2005).

[81] B. A. Kniehl, G. Kramer, I. Schienbein, and H. Spiesberger, Inclusive Charmed-Meson production at the CERN LHC, Eur. Phys. J. C 72, 2082 (2012).

[82] B. A. Kniehl, G. Kramer, I. Schienbein, and H. Spiesberger, Inclusive B-meson production at the LHC in the GM-VFN scheme, Phys. Rev. D 84, 094026 (2011).

[83] B. A. Kniehl, G. Kramer, I. Schienbein, and H. Spiesberger, Inclusive $B$-meson production at small $p_{T}$ in the generalmass variable-flavor-number scheme, Eur. Phys. J. C 75, 140 (2015).

[84] J. Alwall, R. Frederix, S. Frixione, V. Hirschi, F. Maltoni, O. Mattelaer, H. S. Shao, T. Stelzer, P. Torrielli, and M. Zaro, The automated computation of tree-level and nextto-leading order differential cross sections, and their matching to parton shower simulations, J. High Energy Phys. 07 (2014) 079.

[85] M. Cacciari, M. Greco, and P. Nason, The P(T) spectrum in heavy flavor hadroproduction, J. High Energy Phys. 05 (1998) 007.

[86] M. Cacciari, S. Frixione, and P. Nason, The p(T) spectrum in heavy flavor photoproduction, J. High Energy Phys. 03 (2001) 006.

[87] M. Cacciari, S. Frixione, N. Houdeau, M. L. Mangano, P. Nason, and G. Ridolfi, Theoretical predictions for charm and bottom production at the LHC, J. High Energy Phys. 10 (2012) 137.

[88] C. H. Kom, A. Kulesza, and W. J. Stirling, Pair Production of J/psi as a Probe of Double Parton Scattering at LHCb, Phys. Rev. Lett. 107, 082002 (2011).

[89] J.-P. Lansberg and H.-S. Shao, J/psi -pair production at large momenta: Indications for double parton scatterings and large $\alpha_{s}^{5}$ contributions, Phys. Lett. B 751, 479 (2015).

[90] J.-P. Lansberg and H.-S. Shao, Double-quarkonium production at a fixed-target experiment at the LHC (AFTER@LHC), Nucl. Phys. B900, 273 (2015).

[91] H.-S. Shao and Y.-J. Zhang, Complete Study of Hadroproduction of a $\Upsilon$ Meson Associated with a Prompt $J / \psi$, Phys. Rev. Lett. 117, 062001 (2016).

[92] C. Borschensky and A. Kulesza, Double parton scattering in pair production of $J / \psi$ mesons at the LHC revisited, Phys. Rev. D 95, 034029 (2017).

[93] H.-S. Shao and Y.-J. Zhang, Triple Prompt $J / \psi$ Hadroproduction as a Hard Probe of Multiple-Parton Scatterings, Phys. Rev. Lett. 122, 192002 (2019).

[94] K. J. Eskola, I. Helenius, P. Paakkinen, and H. Paukkunen, A QCD analysis of $\mathrm{LHCb}$ D-meson data in $\mathrm{p}+\mathrm{Pb}$ collisions, J. High Energy Phys. 05 (2020) 037.

[95] I. Helenius and H. Paukkunen, Revisiting the D-meson hadroproduction in general-mass variable flavor number scheme, J. High Energy Phys. 05 (2018) 196.

[96] R. Aaij et al. (LHCb Collaboration), Study of prompt $\mathrm{D}^{0}$ meson production in $p \mathrm{~Pb}$ collisions at $\sqrt{s_{\mathrm{NN}}}=5 \mathrm{TeV}$, J. High Energy Phys. 10 (2017) 090.

[97] W. T. Giele and S. Keller, Implications of hadron collider observables on parton distribution function uncertainties, Phys. Rev. D 58, 094023 (1998).
[98] R. D. Ball, V. Bertone, F. Cerutti, L. Del Debbio, S. Forte, A. Guffanti, J. I. Latorre, J. Rojo, and M. Ubiali (NNPDF Collaboration), Reweighting NNPDFs: The W lepton asymmetry, Nucl. Phys. B849, 112 (2011); B855, 927(E) (2012).

[99] R. D. Ball, V. Bertone, F. Cerutti, L. Del Debbio, S. Forte, A. Guffanti, N. P. Hartland, J. I. Latorre, J. Rojo, and M. Ubiali, Reweighting and unweighting of parton distributions and the LHC W lepton asymmetry data, Nucl. Phys. B855, 608 (2012).

[100] N. Sato, J. F. Owens, and H. Prosper, Bayesian reweighting for global fits, Phys. Rev. D 89, 114020 (2014).

[101] H. Paukkunen and P. Zurita, PDF reweighting in the Hessian matrix approach, J. High Energy Phys. 12 (2014) 100 .

[102] A. Kusina, F. Lyonnet, D. B. Clark, E. Godat, T. Jezo, K. Kovarik, F. I. Olness, I. Schienbein, and J. Y. Yu, Vector boson production in $\mathrm{pPb}$ and $\mathrm{PbPb}$ collisions at the $\mathrm{LHC}$ and its impact on nCTEQ15 PDFs, Eur. Phys. J. C 77, 488 (2017).

[103] J. Pumplin, D. Stump, R. Brock, D. Casey, J. Huston, J. Kalk, H. Lai, and W. Tung, Uncertainties of predictions from parton distribution functions. 2. The Hessian method, Phys. Rev. D 65, 014013 (2001).

[104] R. Vogt, Shadowing and absorption effects on J/psi production in dA collisions, Phys. Rev. C 71, 054902 (2005).

[105] C. Lourenco, R. Vogt, and H. K. Woehri, Energy dependence of $\mathrm{J} / \mathrm{psi}$ absorption in proton-nucleus collisions, J. High Energy Phys. 02 (2009) 014.

[106] B. B. Abelev et al. (ALICE Collaboration), Measurement of Prompt $D$-Meson Production in $p-\mathrm{Pb}$ Collisions at $\sqrt{s_{\mathrm{NN}}}=5.02 \mathrm{TeV}$, Phys. Rev. Lett. 113, 232301 (2014).

[107] J. Adam et al. (ALICE Collaboration), Rapidity and transverse-momentum dependence of the inclusive $J / \psi$ nuclear modification factor in $\mathrm{p}-\mathrm{Pb}$ collisions at $\sqrt{s_{\mathrm{NN}}}=5.02 \mathrm{TeV}$, J. High Energy Phys. 06 (2015) 055.

[108] B. B. Abelev et al. (ALICE Collaboration), $J / \psi$ production and nuclear effects in $\mathrm{p}-\mathrm{Pb}$ collisions at $\sqrt{S_{\mathrm{NN}}}=$ 5.02 TeV, J. High Energy Phys. 02 (2014) 073.

[109] R. Aaij et al. (LHCb Collaboration), Study of $J / \psi$ production and cold nuclear matter effects in $p \mathrm{~Pb}$ collisions at $\sqrt{s_{\mathrm{NN}}}=5 \mathrm{TeV}$, J. High Energy Phys. 02 (2014) 072 .

[110] R. Aaij et al. (LHCb Collaboration), Prompt and nonprompt $J / \psi$ production and nuclear modification in $p \mathrm{~Pb}$ collisions at $\sqrt{s_{\mathrm{NN}}}=8.16 \mathrm{TeV}$, Phys. Lett. B 774, 159 (2017).

[111] B. B. Abelev et al. (ALICE Collaboration), Production of inclusive $\Upsilon(1 S)$ and $\Upsilon(2 S)$ in $\mathrm{p}-\mathrm{Pb}$ collisions at $\sqrt{s_{\mathrm{NN}}}=5.02 \mathrm{TeV}$, Phys. Lett. B 740, 105 (2015).

[112] ATLAS collaboration, Measurement of $\Upsilon(\mathrm{nS})$ production with $p+\mathrm{Pb}$ collisions at $\sqrt{s_{\mathrm{NN}}}=5.02 \mathrm{TeV}$ and $p p$ collisions at $\sqrt{s}=2.76 \mathrm{TeV}$, Report No. ATLASCONF-2015-050.

[113] R. Aaij et al. (LHCb Collaboration), Study of $\Upsilon$ production and cold nuclear matter effects in $p \mathrm{~Pb}$ collisions at $\sqrt{s_{\mathrm{NN}}}=5 \mathrm{TeV}$, J. High Energy Phys. 07 (2014) 094.

[114] A. Adare et al. (PHENIX Collaboration), Cold Nuclear Matter Effects on $J / \psi$ Yields as a Function of Rapidity and 
Nuclear Geometry in Deuteron-Gold Collisions at $\sqrt{s_{\mathrm{NN}}}=$ $200 \mathrm{GeV}$, Phys. Rev. Lett. 107, 142301 (2011).

[115] A. Adare et al. (PHENIX Collaboration), Transversemomentum dependence of the $J / \psi$ nuclear modification in $d+\mathrm{Au}$ collisions at $\sqrt{s_{\mathrm{NN}}}=200 \mathrm{GeV}$, Phys. Rev. C 87, 034904 (2013).

[116] M. Aaboud et al. (ATLAS Collaboration), Measurement of quarkonium production in proton-lead and proton-proton collisions at 5.02 TeV with the ATLAS detector, Eur. Phys. J. C 78, 171 (2018).

[117] J. Adam et al. (ALICE Collaboration), D-meson production in $p-\mathrm{Pb}$ collisions at $\sqrt{s_{\mathrm{NN}}}=5.02 \mathrm{TeV}$ and in $\mathrm{pp}$ collisions at $\sqrt{s}=7 \mathrm{TeV}$, Phys. Rev. C 94, 054908 (2016).

[118] S. Carrazza, S. Forte, Z. Kassabov, J. I. Latorre, and J. Rojo, An unbiased hessian representation for Monte Carlo PDFs, Eur. Phys. J. C 75, 369 (2015).

[119] mc2hessian package, https://github.com/scarrazza/ mc2hessian.

[120] S. Dulat, T.-J. Hou, J. Gao, M. Guzzi, J. Huston, P. Nadolsky, J. Pumplin, C. Schmidt, D. Stump, and C. P. Yuan, New parton distribution functions from a global analysis of quantum chromodynamics, Phys. Rev. D 93, 033006 (2016).

[121] Y. Feng, J.-P. Lansberg, and J.-X. Wang, Energy dependence of direct-quarkonium production in $p p$ collisions from fixed-target to LHC energies: Complete one-loop analysis, Eur. Phys. J. C 75, 313 (2015).

[122] J. P. Lansberg, QCD corrections to J/psi polarisation in pp collisions at RHIC, Phys. Lett. B 695, 149 (2011).

[123] S. J. Brodsky and J.-P. Lansberg, Heavy-quarkonium production in high energy proton-proton collisions at RHIC, Phys. Rev. D 81, 051502 (2010).

[124] C. Hadjidakis et al., A fixed-target programme at the LHC: Physics case and projected performances for heavy-ion, hadron, spin and astroparticle studies, Phys. Rep. 911, 1 (2021).

[125] L. Massacrier, B. Trzeciak, F. Fleuret, C. Hadjidakis, D. Kikola, J. P. Lansberg, and H. S. Shao, Feasibility studies for quarkonium production at a fixed-target experiment using the LHC proton and lead beams (AFTER@LHC), Adv. High Energy Phys. 2015, 986348 (2015).

[126] J. P. Lansberg, S. J. Brodsky, F. Fleuret, and C. Hadjidakis, Quarkonium physics at a fixed-target experiment using the LHC beams, Few Body Syst. 53, 11 (2012).

[127] S. J. Brodsky, F. Fleuret, C. Hadjidakis, and J. P. Lansberg, Physics opportunities of a fixed-target experiment using the LHC beams, Phys. Rep. 522, 239 (2013).
[128] U. Acharya et al. (PHENIX Collaboration), Measurement of $J / \psi$ at forward and backward rapidity in $p+p, p+\mathrm{Al}$, $p+\mathrm{Au}$, and ${ }^{3} \mathrm{He}+\mathrm{Au}$ collisions at $\sqrt{s_{\mathrm{NN}}}=200 \mathrm{GeV}$, Phys. Rev. C 102, 014902 (2020).

[129] S. Acharya et al. (ALICE Collaboration), Inclusive $J / \psi$ production at forward and backward rapidity in $\mathrm{p}-\mathrm{Pb}$ collisions at $\sqrt{s_{\mathrm{NN}}}=8.16 \mathrm{TeV}$, J. High Energy Phys. 07 (2018) 160.

[130] J.-P. Lansberg and M. A. Ozcelik, Curing the unphysical behavior of NLO quarkonium production at the LHC and its relevance to constrain the gluon PDF at low scales, arXiv:2012.00702.

[131] E. G. Ferreiro, F. Fleuret, J. P. Lansberg, and A. Rakotozafindrabe, Centrality, rapidity and transverse-momentum dependence of cold nuclear matter effects on J/Psi production in $\mathrm{d} \mathrm{Au}, \mathrm{Cu} \mathrm{Cu}$ and $\mathrm{Au} \mathrm{Au}$ collisions at $s(\mathrm{NN})^{* *}(1 / 2)=200-\mathrm{GeV}$, Phys. Rev. C 81, 064911 (2010).

[132] E. G. Ferreiro, F. Fleuret, J. P. Lansberg, N. Matagne, and A. Rakotozafindrabe, Centrality, Rapidity, and TransverseMomentum Dependence of Gluon Shadowing and Antishadowing on $J / \psi$ Production in $d \mathrm{Au}$ Collisions at $\sqrt{s}=200 \mathrm{GeV}$, Few Body Syst. 53, 27 (2012).

[133] LHCb Collaboration, Study of prompt $\mathrm{D}^{0}$ meson production in $p \mathrm{~Pb}$ at $\sqrt{s_{\mathrm{NN}}}=8.16 \mathrm{TeV}$ at $\mathrm{LHCb}$, Report No. LHCb-CONF-2019-004.

[134] S. Acharya et al. (ALICE Collaboration), Measurement of prompt $\mathrm{D}^{0}, \mathrm{D}^{+}, \mathrm{D}^{*+}$, and $\mathrm{D}_{\mathrm{S}}^{+}$production in $\mathrm{p}-\mathrm{Pb}$ collisions at $\sqrt{\mathrm{s}_{\mathrm{NN}}}=5.02 \mathrm{TeV}$, J. High Energy Phys. 12 (2019) 092.

[135] R. Aaij et al. (LHCb Collaboration), Measurement of $B^{+}$, $B^{0}$ and $\Lambda_{b}^{0}$ production in $p \mathrm{~Pb}$ collisions at $\sqrt{s_{\mathrm{NN}}}=$ 8.16 TeV, Phys. Rev. D 99, 052011 (2019).

[136] S. Acharya et al. (ALICE Collaboration), $\Upsilon$ production in $\mathrm{p}-\mathrm{Pb}$ collisions at $\sqrt{s_{\mathrm{NN}}}=8.16 \mathrm{TeV}$, Phys. Lett. B 806, 135486 (2020).

[137] A. M. Sirunyan et al. (CMS Collaboration), Constraining Gluon Distributions in Nuclei Using Dijets in ProtonProton and Proton-Lead Collisions at $\sqrt{s_{\mathrm{NN}}}=5.02 \mathrm{TeV}$, Phys. Rev. Lett. 121, 062002 (2018).

[138] K. J. Eskola, P. Paakkinen, and H. Paukkunen, Nonquadratic improved Hessian PDF reweighting and application to CMS dijet measurements at 5.02 TeV, Eur. Phys. J. C 79, 511 (2019).

[139] R. A. Khalek et al., Phenomenology of NNLO jet production at the LHC and its impact on parton distributions, Eur. Phys. J. C 80, 797 (2020). 